\title{
Automated approach to measure stellar inclinations: validation through large-scale measurements on the red giant branch ${ }^{\star}$
}

\author{
C. Gehan ${ }^{1}$, B. Mosser², E. Michel ${ }^{2}$, and M. S. Cunha ${ }^{1,3}$ \\ ${ }^{1}$ Instituto de Astrofísica e Ciências do Espaco, Universidade do Porto, CAUP, Rua das Estrelas, 4150-762 Porto, Portugal \\ e-mail: charlotte.gehan@astro.up.pt \\ 2 LESIA, Observatoire de Paris, Université PSL, CNRS, Sorbonne Université, Université de Paris, 5 place Jules Janssen, \\ 92195 Meudon, France \\ 3 School of Physics and Astronomy, University of Birmingham, Birmingham B15 2TT, UK
}

Received 28 August 2020 / Accepted 2 November 2020

\begin{abstract}
Context. Measuring stellar inclinations is fundamental to understanding planetary formation and dynamics as well as the physical conditions during star formation. Oscillation spectra of red giant stars exhibit mixed modes that have both a gravity component from the radiative interior and a pressure component from the convective envelope. Gravity-dominated $(g-m)$ mixed modes split by rotation are well separated inside frequency spectra, allowing accurate measurement of stellar inclinations.

Aims. We aim to develop an automated and general approach to measuring stellar inclinations that can be applied to any solar-type pulsator for which oscillation modes are identified. We also aim to validate this approach using red giant branch stars observed by Kepler.

Methods. Stellar inclination impacts the visibility of oscillation modes with azimuthal orders $m=\{-1,0,+1\}$. We used the mean height-to-background ratio of dipole mixed modes with different azimuthal orders to measure stellar inclinations. We recovered the underlying statistical distribution of inclinations in an unbiased way using a probability density function for the stellar inclination angle.

Results. We derive stellar inclination measurements for 1139 stars on the red giant branch for which Gehan et al. (2018, A\&A, 616, A24) identified the azimuthal order of dipole $g-m$ mixed modes. Raw measured inclinations exhibit strong deviation with respect to isotropy which is expected for random inclinations over the sky. When taking uncertainties into account, the reconstructed distribution of inclinations actually follows the expected isotropic distribution of the rotational axis.

Conclusions. This work highlights the biases that affect inclination measurements and provides a way to infer their underlying statistical distribution. When a star is seen either pole on or equator on, measurements are challenging and result in a biased distribution. Correcting biases that appear in low- and high-inclination regimes allows us to recover the underlying inclination distribution.
\end{abstract}

Key words. asteroseismology - methods: data analysis - techniques: photometric - stars: interiors - stars: low-mass stars: solar-type

\section{Introduction}

The ultra-high-precision photometry space mission Kepler has recorded very long observation runs, providing us with seismic data of unprecedented quality (Gilliland et al. 2010). Red giants represent an ideal laboratory to study the physical mechanisms governing deep stellar interiors because their oscillation spectra exhibit mixed modes. These modes result from a coupling between pressure $(p)$ waves in the convective envelope and gravity $(g)$ waves in the radiative interior and allow us to probe the highly condensed core of red giants (Scuflaire 1974).

Dipole mixed modes present the strongest coupling between $p$ and $g$ waves, as the evanescent region that separates $p$-mode and $g$-mode cavities is thinner compared to mixed modes with higher angular degrees. Dipole mixed modes are therefore the most suitable mixed modes with which to probe the core of red giants (Deheuvels et al. 2017), and have already been used to

\footnotetext{
* The inclination angle of the rotation axis data is only available at the CDS via anonymous ftp to cdsarc.u-strasbg. fr (130.79.128.5) or via http://cdsarc.u-strasbg.fr/viz-bin/cat/J/A+A/645/ A124
}

derive large-scale measurements of several parameters characterising the red giant interior:

- The period spacing between consecutive pure g modes, $\Delta \Pi_{1}$, provides information on the size of the radiative core (Montalbán et al. 2013) and was measured for almost 5000 stars both on the red giant branch (RGB) and in the red clump (Vrard et al. 2016).

- The mean core rotational splitting, $\delta v_{\text {rot,core }}$, is a proxy of the mean core rotation rate and was measured for almost 900 stars on the RGB (Gehan et al. 2018) and 200 stars in the red clump (Mosser et al. 2012a).

- The coupling factor, $q$, characterises the strength of the coupling in the evanescent region between $p$ and $g$ waves and was measured for about 5000 RGB and red clump stars (Mosser et al. 2017a; Pinçon et al. 2020).

- The gravity offset, $\varepsilon_{\mathrm{g}}$, provides information on the stratification occurring in the radiative region and was measured for almost 400 stars both on the RGB and in the red clump (Mosser et al. 2018; Pinçon et al. 2019).

Additionally, dipole mixed modes can also be used to derive high-precision measurements of the stellar inclination angle $i$, which is the angle between the stellar rotation axis and the line of 
sight that take values between $0^{\circ}$ and $90^{\circ}$. Characterising stellar inclinations is important when studying both planetary dynamics and star formation.

On the one hand, in the case of planet host stars, measurements of $i$ are required to constrain the angle between the stellar spin axis and the planetary orbit axis, namely the obliquity $\psi$ (e.g. Winn \& Fabrycky 2015; Chaplin et al. 2013; Huber et al. 2013a; Campante et al. 2016), through Fabrycky \& Winn (2009)

$\cos \psi=\sin i \sin i_{\mathrm{p}} \cos \lambda+\cos i \cos i_{\mathrm{p}}$,

where $i_{\mathrm{p}}$ is the inclination angle of the planetary orbit and $\lambda$ is the sky-projected spin-orbit angle. The angle $i_{\mathrm{p}}$ can be constrained from the transit light curve (see Huber et al. 2018). The angle $\lambda$ can be measured through high-resolution spectroscopic observations of transiting planetary systems, using the so-called Rossiter-McLaughlin effect (Holt 1893; McLaughlin 1924; Rossiter 1924; Queloz et al. 2000; Ohta et al. 2005). The measurement of $i$ is thus a prerequisite to the measurement of the real spin-orbit angle $\psi$, (Kamiaka et al. 2018), which can lead to a better understanding of the formation and evolution of exoplanetary systems. Measuring obliquities helps in particular to constrain the mechanism driving the post-formation migration of hot Jupiters closer to their host star, which is still raising questions (Lin et al. 1996; Chatterjee et al. 2008; Fabrycky \& Tremaine 2007). Indeed, observations indicate that many hot Jupiters show a wide range of obliquities (Johnson et al. 2009; Winn et al. 2010) that can result from planet-star tidal interactions: the stronger the tidal interaction between the star and the planet, the lower the obliquity (Albrecht et al. 2012). However, other scenarios may also result in such high obliquities, which may in this case be observed for different types of planetary systems and not only for hot Jupiters. In this context, measuring stellar obliquities in multiplanet systems is key to testing whether or not spin-orbit misalignment is common (Albrecht et al. 2013). If these systems mostly present low obliquities, then the high obliquities measured for hot Jupiters might be due to planet migration mechanisms. On the contrary, a distribution of obliquities that appears to be similar to that of hot Jupiters would indicate that high obliquities result from formation processes that are common to planetary systems in general, and thus not specific to hot Jupiter migration. Asteroseismology is highly valuable in this context. Indeed, seismic inclinations do not depend on planet size and can therefore be measured for multiplanet systems with small planets, where Rossiter-McLaughlin measurements are usually not possible (see Huber et al. 2018). Asteroseismology has already provided an example of spin-orbit misalignment in a multiplanet system (Huber et al. 2013b).

On the other hand, the statistical distribution of stellar inclinations provides clues as to the physical conditions during star formation (e.g. McKee \& Ostriker 2007). Theoretical models suggest that the angular momentum of forming stars is dominated by small-scale turbulence (e.g. Rey-Raposo \& Read 2018). If some stellar-spin alignment occurs during star formation, one might expect it to be erased by turbulence in the absence of important interactions between forming stars. For a given set of stars with randomly oriented rotation axes, inclination angles follow a distribution in $\sin (i)$, which we refer to as an isotropic inclination distribution in this study. For a given stellar population, an isotropic inclination distribution indicates that turbulence during star formation was strong enough to prevent any stellar spin alignment. On the contrary, a non-isotropic distribution highlights that stellar spins show preferential orientations, and that strong interactions with the progenitor cloud(s) occurred during star formation. Corsaro et al. (2017; C17 hereafter) reported stellar spin alignment towards low inclinations inside two open clusters, however the findings of Mosser et al. (2018) contradict this result. This point is therefore under debate, and inclination measurements can help to discriminate among star formation mechanisms inside open clusters.

The majority of transiting exoplanets are detected around $\mathrm{F}, \mathrm{G}$, and $\mathrm{K}$ solar-type stars on the main sequence, but measuring $i$ is difficult in this evolutionary stage because oscillations have low amplitudes and modes are sometimes blended (see Appourchaux et al. 2008). In this context, Kamiaka et al. (2018) carried out a systematic verification of the accuracy of the seismic measurement of the stellar inclination angle for main sequence stars using 3000 simulated power spectra and found that reliable seismic inclination measurements are possible for $20^{\circ} \lesssim i \lesssim 80^{\circ}$.

However, accurate measurements of $i$ are much easier to obtain for evolved stars presenting mixed modes: $g$-dominated $(g-m)$ mixed modes have lifetimes of the order of years and probe regions that rotate faster than the envelope (Beck et al. 2012; Deheuvels et al. 2012, 2014, 2015; Benomar et al. 2013; Mosser et al. 2017a). Therefore, rotational splittings are much larger than mode line widths and dipole modes split by rotation are well separated. Kuszlewicz et al. (2019a; K19 hereafter) recently developed a Bayesian hierarchical method to extract inclination angles from red giants and successfully applied it to artificial oscillation spectra. They also derived inclination measurements for about 100 red giant stars. As Kamiaka et al. (2018) did not address red giants and K19 measured inclinations for only a limited number of red giants, it is worthwhile obtaining large-scale measurements of the stellar inclination angle for evolved stars.

Nevertheless, it is difficult to detect exoplanetary transits around evolved stars because the associated depth in the light curve varies as the squared ratio between planet and star radii, $\left(R_{\mathrm{p}} / R_{\star}\right)^{2}$ (Heller 2019). The Kepler mission is optimised to detect Earth-size planets in the habitable zone of solar-like stars, and can therefore detect a depth of $0.01 \%$ in relative stellar brightness fluctuations, which corresponds to an Earth-sized planet transiting a Sun-like star with $R_{\mathrm{p}} \sim R_{\star} / 100$. Red giants have a mean radius on the order of $R_{\star} \sim 10 R_{\odot}$, and therefore the transit of an Earth-sized planet leads to a depth of only $10^{-6}$ in relative brightness. If we consider a Jupiter-like planet with a radius $R_{\mathrm{p}} \sim 10 R_{\oplus}$, where $R_{\oplus}$ is the Earth's radius, the transit in front of a red giant corresponds to a depth of $0.01 \%$ in relative brightness, which is the same order of magnitude as an Earth-sized planet transiting a Sun-like star. The detection of hot Jupiters transiting red giant stars is therefore possible, in contrast to transiting Earth-size planets.

About 100 exoplanets are already known to orbit red giants and subgiants (Johnson et al. 2010, 2011; Hirano et al. 2015; Lillo-Box et al. 2014, 2016; Jones et al. 2015; Lee et al. 2015; Quinn et al. 2015; Hrudková et al. 2017). The detection of a much larger number of planets around evolved stars is crucial to constrain theoretical models of planet engulfment by the expanding host star (Lillo-Box et al. 2016), and to understand the effect of stellar evolution on the orbital and physical properties of planetary systems (Jones et al. 2015). In this context, measuring stellar inclination angles on a large scale for evolved stars is a major stepping stone in understanding planetary formation, evolution, and death.

The method presented in Gehan et al. (2018) identifies rotational splittings of $g-m$ modes for stars on the RGB. It is therefore possible to measure the stellar inclination angle using the 
ratio of the power spectral densities (PSDs) of dipole mixed modes with the same mixed-mode order but different azimuthal orders (Gizon \& Solanki 2003). In this work we present a general and automated method to derive seismic measurements of the stellar inclination angle that can be applied to any solar-type pulsator for which oscillation modes are identified. We check the consistency of our approach on a large sample of red giant stars, from which we should recover an isotropic inclination distribution. In Sect. 2 we provide details of the way in which we derive stellar inclinations and their associated uncertainties. In Sect. 3 we apply the method to the RGB stars of the Kepler public catalogue studied by Gehan et al. (2018) and infer the underlying statistical distribution of the measured inclinations. In Sects. 4 and 5 we check the consistency of our results with those obtained by K19 and C17. Section 6 focuses on a discussion of the inclinations measured for RGB stars with a planet candidate(s) or a confirmed planet(s). We present our conclusions in Sect. 7.

\section{Deriving stellar inclinations}

Measuring stellar inclinations using frequency oscillation spectra is not straightforward and requires several steps.

\subsection{Disentangling mixed modes with different azimuthal orders}

The identification of dipole mixed modes with different azimuthal orders relies on oscillation spectra in stretched period, where radial and quadrupole modes have been removed using the red giant universal oscillation pattern which characterises pressure modes (Mosser et al. 2011). This stretching is done using the function

$\zeta=\left[1+\frac{v^{2}}{q} \frac{\Delta \Pi_{1}}{\Delta v_{\mathrm{p}}} \frac{1}{\frac{1}{q^{2}} \sin ^{2}\left(\pi \frac{\nu-v_{\mathrm{p}}}{\Delta v_{\mathrm{p}}}\right)+\cos ^{2}\left(\pi \frac{\nu-v_{\mathrm{p}}}{\Delta v_{\mathrm{p}}}\right)}\right]^{-1}$,

which characterises the contribution of the core and envelope to the inertia of dipole modes (Goupil et al. 2013; Mosser et al. 2015; Hekker \& Christensen-Dalsgaard 2017; Cunha et al. 2019). The $\zeta$ function as expressed in Eq. (2) is continuous with frequency $v$ and depends on:

- the coupling parameter $q$ between $g$ and $p$ modes (Mosser et al. 2017b);

- the asymptotic large separation,

$\Delta v_{\mathrm{p}}=\Delta v\left(1+\alpha\left(n_{\mathrm{p}}-n_{\max }\right)\right)$,

where $n_{\max }=v_{\max } / \Delta v-\varepsilon_{\mathrm{p}}$ is the non-integer order at the frequency $v_{\max }$ of maximum oscillation signal, with $\varepsilon_{\mathrm{p}}$ the phase shift of pure pressure modes, and where $\Delta v_{\mathrm{p}}$ increases with the pressure radial order $n_{\mathrm{p}}$ (Mosser et al. 2013);

- and the pure dipole pressure mode frequencies, $v_{\mathrm{p}}$, computed using the universal pattern (Mosser et al. 2011).

Stretched-period spectra are then obtained using the differential equation (Mosser et al. 2015)

$\mathrm{d} \tau=\frac{1}{\zeta} \frac{\mathrm{d} v}{v^{2}}$.

Dipole mixed modes are expected to be evenly spaced in stretched period with a spacing equal to $\Delta \Pi_{1}$, as in pure dipole $g$ modes. However, $p$-dominated $(p-m)$ dipole mixed modes present large line widths and consequently appear as several modes scattered close to the expected unique $p-m$ mode. This spread is exacerbated when stretching frequency spectra, as the correction induced by $\zeta$ is most important for $p$ modes (Gehan et al. 2018). As $p$ - $m$ dipole mixed modes scramble the expected regular pattern in stretched period, we remove them from stretched-period spectra using once again the red giant universal pattern (Mosser et al. 2011). We are now left with $g$ - $m$ dipole modes only, which should in theory present an equal $\Delta \Pi_{1}$ spacing in stretched period. However, rotation associated to the $g$-mode cavity disturbs this regular pattern, meaning that the stretched period spacing between modes with the same azimuthal order is (Mosser et al. 2015)

$\Delta \tau_{\mathrm{m}} \simeq \Delta \Pi_{1}\left(1+2 m \zeta \frac{\delta v_{\text {rot,core }}}{v}\right)$.

In this equation, $\zeta$ can be estimated for $g$ - $m$ mixed modes as $\mathcal{N} /(\mathcal{N}+1)$, where $\mathcal{N}$ is the mixed-mode density representing the number of $g$ modes per $\Delta v$-wide frequency range and is defined as

$\mathcal{N}=\frac{\Delta v}{\Delta \Pi_{1} v_{\max }^{2}}$

Mixed modes with different azimuthal orders can now be easily disentangled using stretched period échelle diagrams (Mosser et al. 2015, 2018; Gehan et al. 2018), where mixed modes with the same azimuthal order draw ridges called rotational components (Fig. 1). Peaks associated to the $g-m$ modes that have a maximum PSD significantly above the background are kept (see Sect. 2.2 of Gehan et al. 2018), and their maximum PSD is used in the following. The number of visible ridges depends on the stellar inclination angle, which modifies the visibility of the different rotational components: when the inclination is low, only the central rotational component associated to $m=0$ is visible, for intermediate $i$ values all the three ridges with $m=\{-1,0,+1\}$ are visible, and in the case of high $i$ values, only the $m= \pm 1$ components are visible. Each ridge with a given azimuthal order is identified through a correlation of the observed spectrum with a synthetic one built upon asymptotic seismic parameters using Eqs. (5) and (6), as described by Gehan et al. (2018). The azimuthal order of each $g-m$ mode is thus identified, making it possible to measure the stellar inclination angle.

\subsection{Measurement of the stellar inclination angle}

We now detail how we measure the stellar inclination angle $i$. We write $p_{-1}(v), p_{0}(v)$, and $p_{1}(v)$, the PSD of individual $g$ - $m$ modes with azimuthal orders $m=\{-1,0,+1\}$, respectively. The PSD is related to the stellar inclination by (Gizon \& Solanki 2003)

$p_{0}(v) \propto \cos ^{2}(i)$

$p_{ \pm 1}(v) \propto \frac{1}{2} \sin ^{2}(i)$

The background signal close to $v_{\max }$ can be approximated by

$B(v)=\beta\left(\frac{v}{v_{\max }}\right)^{\gamma}$,

where $\beta$ and $\gamma$ are parameters that take real values (Mosser et al. $2012 b$ ). This expression is valid in the frequency range where oscillations are detected, because it provides the precise measurement of $v_{\max }$ (Fig. 8 of Pinsonneault et al. 2018). 


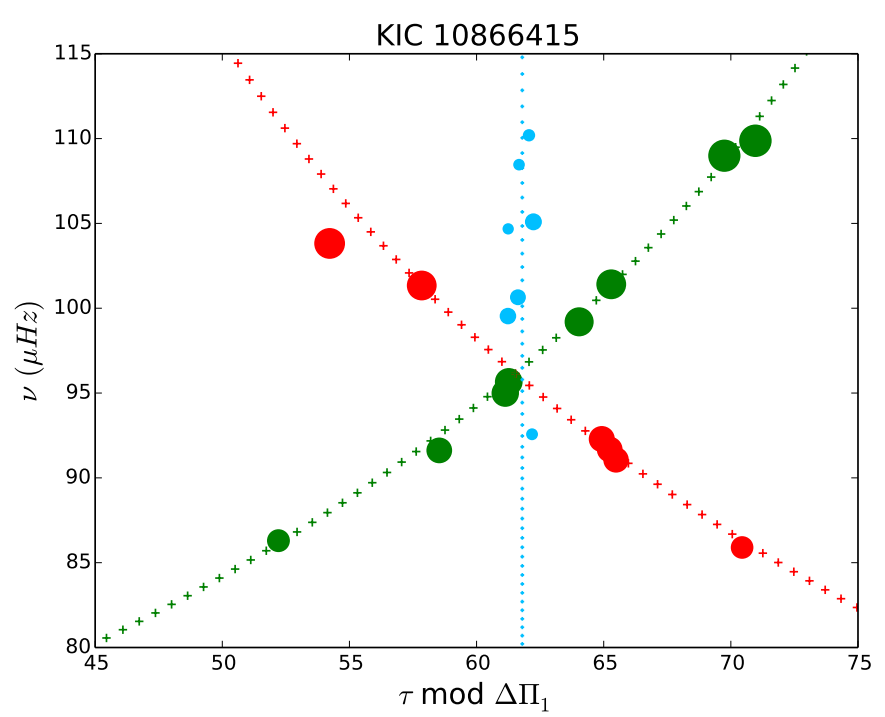

Fig. 1. Stretched-period échelle diagram for the RGB star KIC 10866415 with a high inclination angle. Colours indicate the azimuthal order: the $m=\{-1,0,+1\}$ rotational components are represented in green, light blue, and red, respectively. Observed modes are represented by dots and the symbol size varies as a function of the measured power spectral density. Rotational components with $m= \pm 1$ are identified in an automatic way through a correlation of the observed spectrum with a synthetic one constructed using Eqs. (5) and (6), represented here by crosses (Gehan et al. 2018). The location of the $m=0$ rotational component is identified considering that the $m=0$ ridge is median with respect to $m= \pm 1$ ridges. As there are six modes identified in the $m=+1$ ridge and eight modes in the $m=-1$ ridge, this gives a mean number of visible modes $N_{ \pm 1}=7$. We thus consider the first seven modes with the highest height-to-background ratio belonging to the $m=0$ ridge.

We then subtract the background from the PSD of each mode and normalise the result by $B(v)$. We thus consider the background-free and normalised PSD for each mode of azimuthal order $m$, which we denote

$\mathcal{P}_{\mathrm{m}}(v)=\frac{p_{\mathrm{m}}(v)}{B(v)}-1$.

As the height-to-background ratio (HBR) of modes is supposed to follow a Gaussian envelope centred on $v_{\max }$, we then weight the contribution of modes with same azimuthal order by a Gaussian function denoted $f_{\mathrm{G}}(v)$. This step maximises the contribution of modes in the close vicinity of $v_{\max }$, which are expected to have the highest HBR, and minimises the contribution of modes in the tails of the Gaussian envelope, which are expected to have the lowest HBR. The standard deviation of the Gaussian function can be written (Mosser et al. 2012b)

$\sigma=\frac{\delta v_{\mathrm{env}}}{2 \sqrt{2 \ln 2}}$

where $\delta v_{\text {env }}$ is a frequency interval chosen to be slightly larger than the frequency range of observed dipole modes. We write the weighting of $\mathcal{P}_{\mathrm{m}}(v)$ (Eq. (9)) by the Gaussian function $f_{\mathrm{G}}(v)$ as

$P_{\mathrm{m}}(v)=\mathcal{P}_{\mathrm{m}}(v) f_{\mathrm{G}}(v)$.

We then consider the mean value of $P_{\mathrm{m}}(v)$ in Eq. (11) over all $n$ modes with a given azimuthal order such as

$\left\langle P_{\mathrm{m}}\right\rangle=\frac{\sum_{\mathrm{i}=1}^{n} P_{\mathrm{m}, \mathrm{i}}(v)}{\sum_{\mathrm{i}=1}^{n} f_{\mathrm{G}, \mathrm{i}}(v)}$.

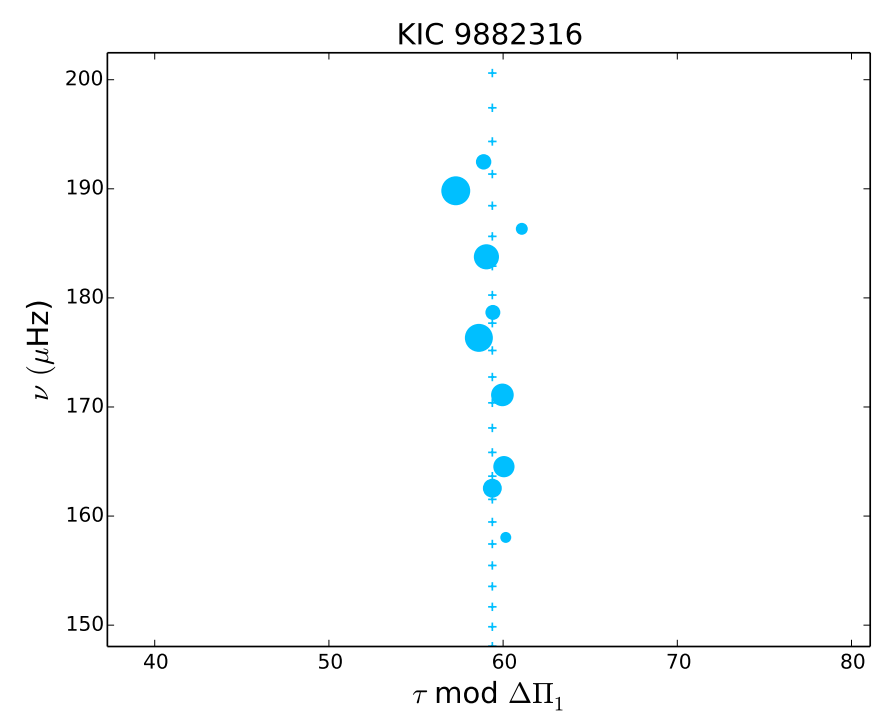

Fig. 2. Same as Fig. 1 but for the RGB star KIC 9882316 with a low inclination angle.

We can finally measure $i$ from Eq. (7) through

$\tan (i)=\sqrt{\frac{\left\langle P_{1}\right\rangle+\left\langle P_{-1}\right\rangle}{\left\langle P_{0}\right\rangle} .}$

\subsubsection{Intermediate inclinations}

When all the three rotational components associated to $m=$ $\{-1,0,+1\}$ are visible, we can directly derive a measurement of the inclination angle using Eq. (13).

\subsubsection{Low inclinations}

Measuring low inclinations is particularly tricky, and we can only derive a rough estimate of $i$. Indeed, when the stellar inclination is low, the PSD of mixed modes associated to $m= \pm 1$ is so low that these modes are lost in the background and are no longer visible (Fig. 2). We do not know where we can find the missing $m= \pm 1$ components in the signal; we can only make the assumption that non-visible modes have a HBR below a given threshold $x$, corresponding to $\mathcal{P}_{ \pm 1}(v)=x-1$ in Eq. (9). In the absence of direct information on the HBR of the $m= \pm 1$ components, we suppose that they are symmetric and have similar $x$ values. Using Eq. (12), we thus have an upper limit $i_{\max }$ for low stellar inclinations, such as

$\tan (i)<\sqrt{\frac{2\left\langle P_{ \pm 1}\right\rangle}{\left\langle P_{0}\right\rangle}}=\tan \left(i_{\max }\right)$.

Estimating the PSD of the missing $m= \pm 1$ modes. We have to provide an estimate of the maximum possible PSD of the missing $m= \pm 1$ modes in order to derive an upper limit on $i$. As in the case of stars presenting two and three rotational components, we consider that the threshold $x$ follows a Gaussian with a standard deviation computed as in Eq. (10), in order to follow the Gaussian shape of the power spectrum around $v_{\max }$ (Fig. 3). In order to chose the maximum value taken by $x$ around $v_{\max }$, we considered the threshold HBR above which $g$ - $m$ modes were considered as significant and were kept to initiate the fit of rotational components in the study of Gehan et al. (2018). Among 


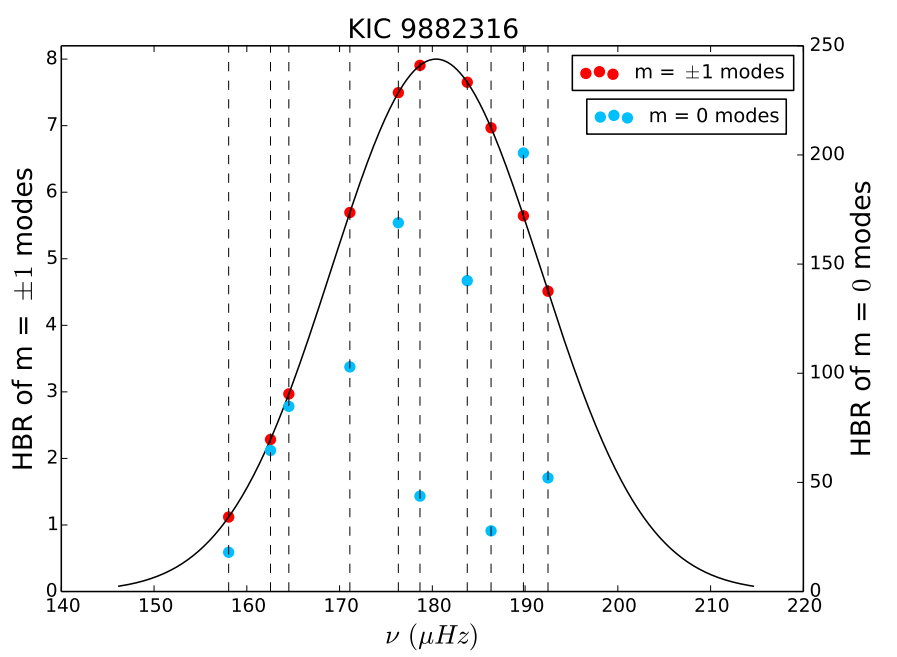

Fig. 3. HBR of $g-m$ modes for KIC 9882316 , for which only the central $m=0$ rotational component is visible. Observed modes with $m=0$ are in light blue (characterised by the right ordinate axis). Missing modes with $m= \pm 1$ are in red (characterised by the left ordinate axis), and their HBR follows a Gaussian envelope (in black) referred to as $x$ in the core text, which is normalised to the value of 8 . Vertical dashed lines identify frequencies of observed $m=0$ modes. The number of missing $m= \pm 1$ modes has been chosen to be the same as visible $m=0$ modes.

the 1139 RGB stars for which Gehan et al. (2018) have identified rotational components, we note that the distribution of the threshold HBR peaks around 8, and that the number of stars in our sample sharply decreases below a threshold HBR of 8 (Fig. 4). This indicates that rotational components can only be poorly identified through the method developed by Gehan et al. (2018) when the threshold HBR drops below 8. We also notice that only three stars in our sample have a threshold HBR that is below 4 (Fig. 4), which is compatible with the minimum HBR of 4 that is found by K19 to reliably extract the inclination angle from their artificial spectra.

According to the distribution of the HBR shown in Fig. 4, we chose a maximum value of $x_{\max }=8$ for the Gaussian that models the HBR of the missing $m= \pm 1$ components (Fig. 3). For cases where the mean HBR is below 8 for modes belonging to the $m=0$ component, we took this mean HBR value as a maximum value for $x_{\max }$. In order to compute $\left\langle P_{ \pm 1}\right\rangle$ in Eq. (14), we estimated the maximum PSD of the missing $m= \pm 1$ modes by interpolating the Gaussian threshold $x$ to the frequencies of observed $m=0$ modes so that we have the same number of $m=0$ and $m= \pm 1$ modes (Fig. 3 ). We then computed $\left\langle P_{ \pm 1}\right\rangle$ using Eq. (12).

The threshold $x_{\max }$ is linked to the total observation duration $T_{\text {obs }}$ such as (Mosser et al. 2018)

$x_{\max } \simeq \ln \left(\frac{T_{\mathrm{obs}} \Delta_{v}}{p}\right)$,

where $\Delta_{v}$ is the width of the frequency range where a mode is expected and $p$ is the rejection probability of the null hypothesis, that is, the hypothesis that background noise alone is enough to explain the presence of the observed peaks. Red giants observed by Kepler have $T_{\text {obs }}=4$ years, and we have $\Delta_{v}=0.1 \mu \mathrm{Hz}$ in the case of $g-m$ modes. The maximum value $x_{\max }=8$ considered in this work corresponds to a rejection probability of the nullhypothesis at the $0.4 \%$ level.

Inclination estimate. When only the $m=0$ rotational component is visible, we can only have an estimate of the inclination

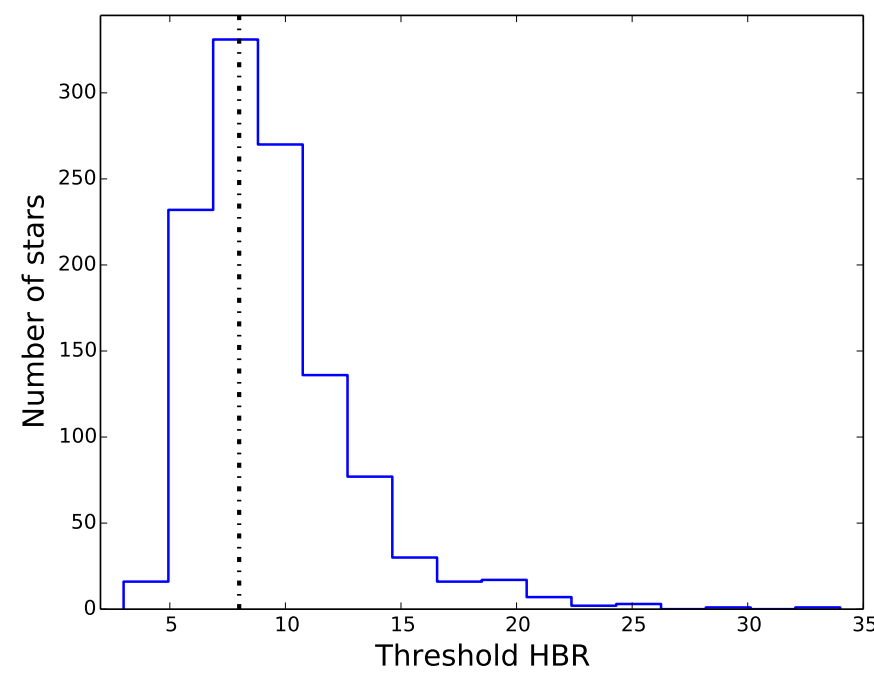

Fig. 4. Histogram of the threshold HBR above which $g$ - $m$ modes were considered as significant and were kept to initiate the fit of rotational components in the study of Gehan et al. (2018), for our sample of 1139 RGB stars. The vertical dot-dashed line indicates a threshold HBR of 8 .

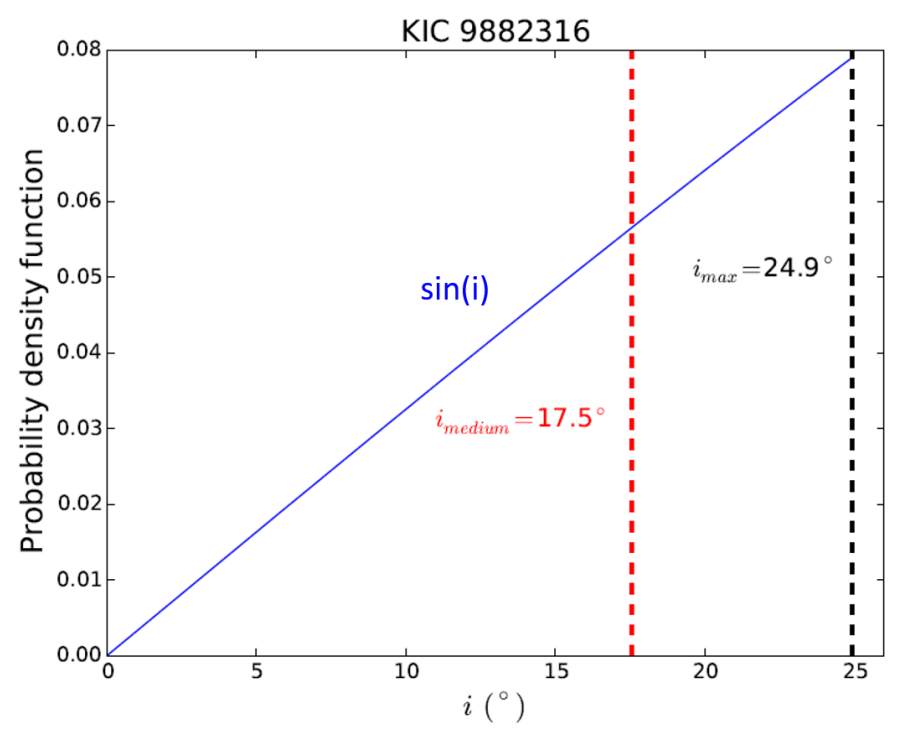

Fig. 5. Probability density function in $\sin (i)$ between $0^{\circ}$ and $i_{\max }$ for KIC 9882316 (in blue). Black dashed lines indicate the upper limit on the inclination derived from Eq. (14), here $i_{\max }=24.9^{\circ}$. Red dashed lines indicate the estimated inclination corresponding to a PDF value of 0.5 , here $i_{\text {medium }}=17.5^{\circ}$.

angle, which can take any value between $0^{\circ}$ and $i_{\max }$ given by Eq. (14). In order to provide an estimate of $i$, rather than just the upper limit $i_{\max }$, we assume for a given star that the possible inclination values are isotropically distributed between $0^{\circ}$ and $i_{\max }$. Under this hypothesis, the probability of having an inclination between $i$ and $i+\mathrm{d} i$, where $\mathrm{d} i$ is an infinitesimal inclination angle, is proportional to the spherical area covered by $\mathrm{d} i$, and therefore to the corresponding specific solid angle $\mathrm{d} \Omega=2 \pi \sin (i) \mathrm{d} i$. We therefore consider that the inclination follows a probability density function (PDF) in $\sin (i)$ between $0^{\circ}$ and $i_{\max }$ (Fig. 5). We then derive an estimate of the mean inclination by selecting the $i$ value corresponding to a PDF value of 0.5 ( $i_{\text {medium }}$ in Fig. 5). 


\subsubsection{High inclinations}

When the inclination is high and only the $m= \pm 1$ components are visible, we can rely on the existing signal to find the missing $m=0$ component. The location of the $m=0$ component is derived considering that the $m=0$ ridge is median with respect to $m= \pm 1$ ridges. We consider, as in the fitting procedure of the rotational components used in Gehan et al. (2018), that peaks belong to the $m=0$ rotational component if they are at a distance of no more than $\Delta \Pi_{1} / 80$ from the mid-point between the $m=-1$ and $m=+1$ components in the stretched-period spectrum. We then compute $N_{ \pm 1}$, the mean number of identified modes with azimuthal orders $m= \pm 1$, and we keep the $N_{ \pm 1}$ modes belonging to the $m=0$ ridge that have the highest HBR estimated using Eq. (9); see Fig. 1. This step allows us to compute $\left\langle P_{0}\right\rangle$ in Eq. (12). We then distinguish between two different configurations, depending on the mean $\operatorname{HBR}\left\langle P_{0}\right\rangle$ of the retrieved $m=0$ component. If $\left\langle P_{0}\right\rangle$ is sufficiently high, then we have clear oscillation modes and we can derive a measurement of the inclination through Eq. (13). On the contrary, if $\left\langle P_{0}\right\rangle$ is below a given threshold, we cannot know if the visible signal is due to oscillation modes or to stochastic variations of the background noise and we can only derive an estimate of the inclination instead of a firm measurement. We consider the threshold value $\left\langle P_{0}\right\rangle=8$ to separate stars with two components for which we obtain an inclination measurement from stars with a $m=0$ component hidden in the background noise, for which we can only derive an estimate of $i$.

\subsection{Estimate of the uncertainties}

Uncertainties on the measured stellar inclinations mainly arise from the stochastic nature of oscillations that is a source of spread in the PSD of observed modes, and therefore in their HBR, impacting the precision of the inclination measurement. Uncertainties are derived from Eq. (13). We can write

$\frac{\mathrm{d} \tan ^{2}(i)}{\tan ^{2}(i)}=\frac{\mathrm{d} P_{+1}}{\left\langle P_{+1}\right\rangle}+\frac{\mathrm{d} P_{-1}}{\left\langle P_{-1}\right\rangle}+\frac{\mathrm{d} P_{0}}{\left\langle P_{0}\right\rangle}$,

where $P_{\mathrm{m}}(v)$ is defined by Eq. (11) and $\left\langle P_{\mathrm{m}}\right\rangle$ is defined by Eq. (12). Moreover, we have

$\frac{\mathrm{d} \tan ^{2}(i)}{\tan ^{2}(i)}=2 \frac{\mathrm{d} \tan (i)}{\tan (i)}=2\left(\frac{1+\tan ^{2}(i)}{\tan (i)}\right) \mathrm{d} i=\frac{4}{\sin (2 i)} \mathrm{d} i$.

The equality of Eqs. (16) and(17) gives

$\mathrm{d} i=\frac{1}{4} \sin (2 i)\left(\frac{\mathrm{d} P_{+1}}{\left\langle P_{+1}\right\rangle}+\frac{\mathrm{d} P_{-1}}{\left\langle P_{-1}\right\rangle}+\frac{\mathrm{d} P_{0}}{\left\langle P_{0}\right\rangle}\right)$.

We finally obtain the uncertainty $\sigma_{\mathrm{i}}$ on the measured inclination through

$\sigma_{\mathrm{i}}=\frac{1}{4}|\sin (2 i)| \sqrt{\sum_{m=-1}^{m=+1}\left(\frac{\Delta P_{\mathrm{m}}}{\left\langle P_{\mathrm{m}}\right\rangle}\right)^{2}}$,

where $\Delta P_{\mathrm{m}}$ is the standard deviation of $P_{\mathrm{m}}(v)$ used in Eq. (11).

Equation (19) provides $1-\sigma$ uncertainties. Error bars are therefore symmetric around the measured inclination value, and we have $\sigma_{i}=\sigma_{i,-}=\sigma_{i,+}$. However error bars should not be symmetric when the inclination is low or high and we should have different lower and upper error bars $\sigma_{i,-}$ and $\sigma_{i,+}$, respectively. For these two extreme cases, uncertainties differ from 1- $\sigma$ uncertainties.

\subsubsection{Low inclinations}

When the $m= \pm 1$ rotational components are missing, the lower error bar $\sigma_{i,-}$ is computed assuming that the lowest possible inclination is $0^{\circ}$. The upper error bar $\sigma_{i,+}$ on the $i_{\max }$ value derived from Eq. (14) corresponds to a $1-\sigma$ uncertainty and is computed using Eq. (19). The upper error bar on $i_{\text {medium }}$ is computed assuming that the highest possible inclination is $i_{\max }+\sigma_{i,+}$.

\subsubsection{High inclinations}

When only the two rotational components with azimuthal orders $m= \pm 1$ are visible, there are two possible configurations (Sect. 2.2.3) impacting the way uncertainties are derived.

For stars with a mean HBR of the $m=0$ rotational component $\left\langle P_{0}\right\rangle \geq 8$, we have a firm measurement of the inclination (Sect. 2.2.3) and we have symmetric error bars corresponding to 1- $\sigma$ uncertainties computed using Eq. (19).

The other configuration corresponds to stars with a mean HBR $\left\langle P_{0}\right\rangle<8$, for which the inclination can in principle be as high as $90^{\circ}$. However, as stressed by Kamiaka et al. (2018), it is in practice impossible to measure $i=90^{\circ}$, as this would require an infinite HBR corresponding to a strict absence of background noise from granulation. Therefore, we cannot access the inclination regime close to $90^{\circ}$. Hence, stars that are in this configuration in our sample should have upper error bars allowing the value $i=90^{\circ}$ to be encompassed as a possible extreme value for the inclination. The upper error bar $\sigma_{i,+}$ for these stars is thus computed assuming that the highest possible inclination is $i=90^{\circ}$. The lower error bar $\sigma_{i,-}$ corresponds to a $1-\sigma$ uncertainty and is computed using Eq. (19).

\section{Results}

We analysed 1139 stars on the $\mathrm{RGB}^{1}$ for which Gehan et al. (2018) identified rotational components; we refer to this paper for the details of the data analysis. The oscillation spectra and the stretched-period échelle diagrams of some of these stars are shown in Appendix B, with the azimuthal order of mixed modes identified. We analyse the distribution of inclinations and test its compatibility with isotropy.

\subsection{Raw stellar inclinations}

When looking at stars randomly selected over a large fraction of the sky, we expect stellar inclinations to be randomly distributed following a $\sin (i)$ distribution. Stars analysed here have been considered following increasing KIC numbers. This is not supposed to induce any selection bias and inclinations derived in this study should be isotropically distributed.

The raw distribution of stellar inclinations of our sample appears to deviate significantly from an isotropic distribution (Fig. 6). This is expected because measuring $i=0$ or $90^{\circ}$ is impossible in practice as explained in Sect. 2.3.2, resulting in a dramatic deficit of values in these extreme inclination regimes. We obtain firm inclination measurements between $27^{\circ}$ and $78^{\circ}$ for stars presenting three rotational components and stars presenting two rotational components for which the mean HBR of the retrieved $m=0$ component is equal to or above 8 (Fig. 6). This is consistent with the conclusion of Kamiaka et al. (2018), namely that reliable seismic inclination measurements are possible in the general case for $20^{\circ} \lesssim i \lesssim 80^{\circ}$. For $i<27^{\circ}$,

\footnotetext{
1 Results are publicly available on ADS.
} 


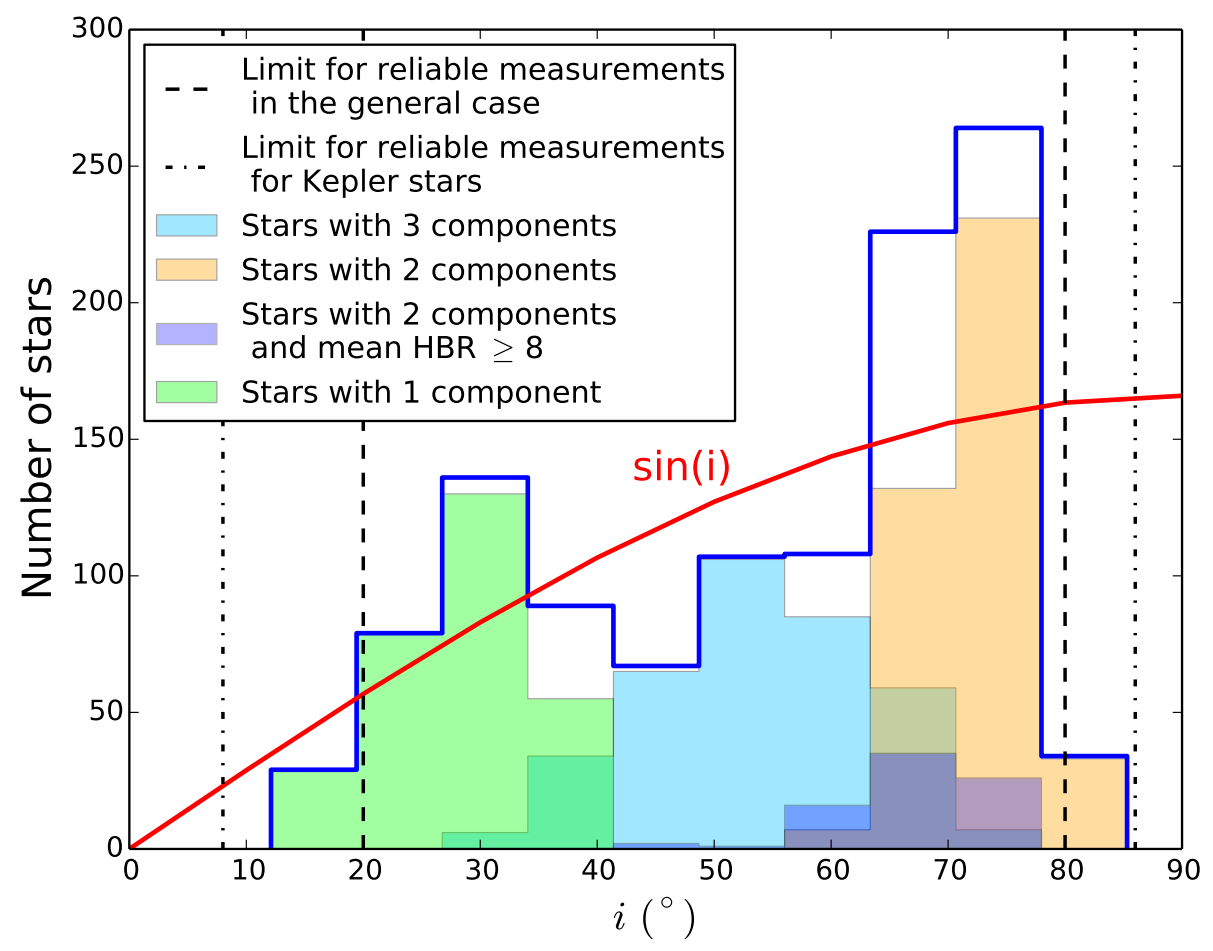

Fig. 6. Distribution of raw stellar inclinations for 1139 RGB stars analysed by Gehan et al. (2018) (in blue). The red line represents an isotropic distribution in $\sin (i)$ derived from a fit to the histogram in blue. Inclinations measured for stars presenting three rotational components are in light blue, while inclination estimates for stars presenting one (two) rotational components are in green (orange). Inclination measurements derived for stars presenting two rotational components and a mean HBR equal to or above 8 for the $m=0$ component are in violet. Vertical dashed lines represent the limits of $20^{\circ}$ and $80^{\circ}$ between which Kamiaka et al. (2018) consider seismic inclination measurements to be reliable in the general case. Vertical dot-dashed lines represent the limits of $8^{\circ}$ and $86^{\circ}$ between which Kamiaka et al. (2018) show that seismic inclination measurements can be reliable for Kepler stars.

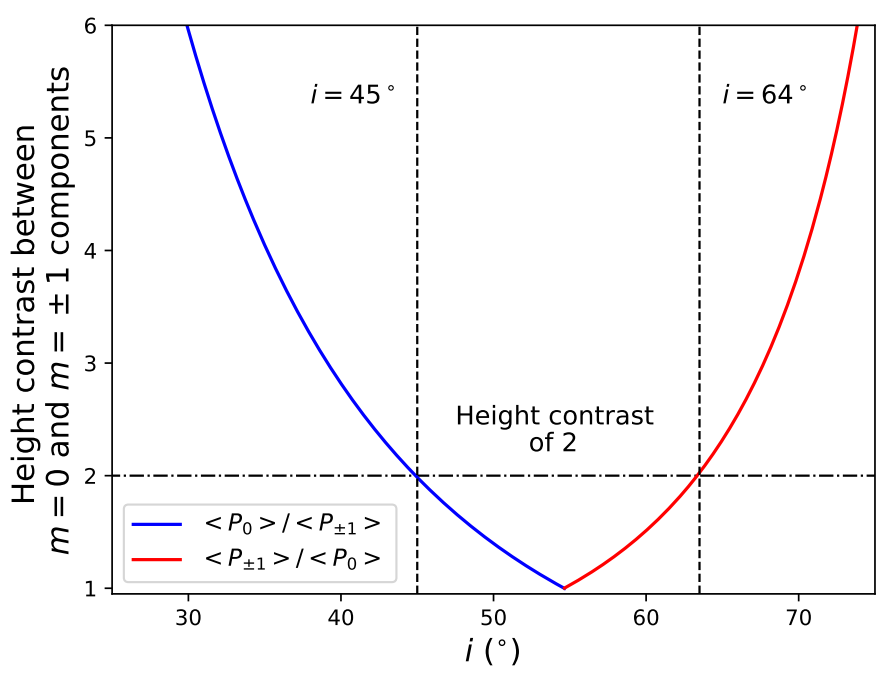

Fig. 7. Evolution with inclination of the height contrast between rotational components with azimuthal orders 0 and \pm 1 . The blue line represents the contrast $\left\langle P_{0}\right\rangle /\left\langle P_{ \pm 1}\right\rangle$ computed from Eq. (12), while the red line represents the contrast $\left\langle P_{ \pm 1}\right\rangle /\left\langle P_{0}\right\rangle$. Vertical dashed lines indicate inclinations of 45 and $64^{\circ}$, while the horizontal dot-dashed line indicates a height contrast of 2 .

we can only derive an overestimated value of the inclination. The same phenomenon occurs at high inclination, where we can only derive an underestimated value of the inclination for $i>77^{\circ}$. We have no inclination estimate below $12^{\circ}$ and above $85^{\circ}$. This is consistent with Fig. 3 of Kamiaka et al. (2018), which shows that reliable inclination measurements are possible between $8^{\circ} \lesssim i \lesssim 86^{\circ}$ for stars with a high HBR of 30 and an observation duration of 4 years. Our results for Kepler red giants confirm that we cannot derive inclination estimates outside of these limit values.

We note that the inclination distribution for stars presenting three rotational components (light blue in Fig. 6) peaks around $55^{\circ}$ and decreases below $45^{\circ}$ and above $64^{\circ}$. This is expected because heights of rotational components with different azimuthal orders are equal at $55^{\circ}$, giving a minimal height contrast between components (Fig. 7). We checked that the values $i=45^{\circ}$ and $64^{\circ}$ correspond to a height ratio between components with different $m$ values that reaches values above approximately 2 (Fig. 7). This highlights the fact that the efficiency of the detection of all the $m$ components decreases when the height contrast between ridges with different azimuthal orders surpasses a certain threshold. For $i$ values above $64^{\circ}$, the non-detected $m=0$ component can be retrieved at the mid-point between $m=-1$ and $m=+1$ components (Sect. 2.3.2 and Fig. 1), allowing us to avoid a drop in measured inclinations in this regime (violet in Fig. 6). However, we miss the $m= \pm 1$ components for stars with $i$ values below $45^{\circ}$. These stars are therefore lost among the population of stars showing only one rotational component, leading to the dip observed in Fig. 6 between $40^{\circ}$ and $50^{\circ}$.

\subsection{Unbiased inclination distribution}

We cannot obtain a firm measurement of the inclination for stars presenting one rotational component or for stars with two components and a low mean HBR for the retrieved $m=0$ component, but only a range of possible values. We take individual uncertainties into account to test the inclination distribution in a statistical sense.

In the particular cases where only one $(m=0)$ or only two ( $m= \pm 1$ ) rotational components are hidden in the background noise, we assume that the inclination is isotropically distributed between the minimum possible value $i_{\min }$ and the maximum possible value $i_{\max }$ for each star. We thus assumed a PDF in $\sin (i)$ for these stars (Fig. 5). Additionally, we assumed a Gaussian PDF for stars presenting three rotational components as well as for stars with two rotational components and a mean HBR equal to or above 8 for the retrieved $m=0$ component. The Gaussian $\mathrm{PDF}$ is centred on the measured inclination value and has a standard deviation equal to $\sigma_{\mathrm{i}} /(2 \sqrt{2 \ln 2})$ (Eq. (10)). By integrating 


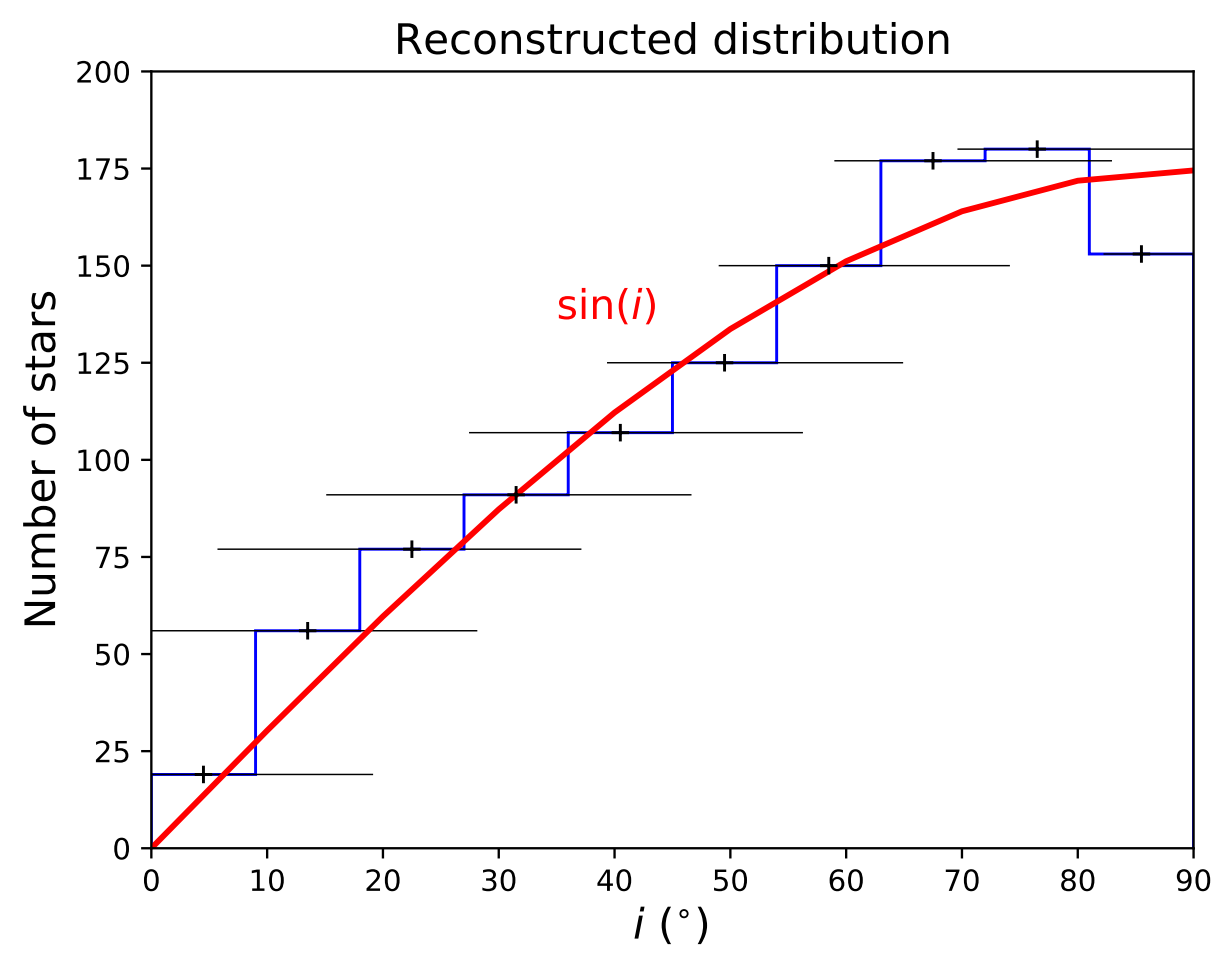

Fig. 8. Reconstructed distribution of stellar inclinations for our sample of 1139 RGB stars. We assumed an isotropic PDF in $\sin (i)$ for all stars presenting one rotational component as well as for stars presenting two rotational components and a $m=0$ component lost in the background. We used a Gaussian PDF for stars presenting three rotational components as well as for stars with two rotational components and a mean HBR equal to or above 8 for the retrieved $m=0$ component. The red line represents an isotropic distribution in $\sin (i)$ derived from a fit to the histogram. Mean error bars are represented in black for each inclination bin. the PDF of all these stars, we retrieve a global distribution of inclinations for which the statistic is free from the observational limitations hampering the measurement of low and high $i$ values.

We obtain a reconstructed global distribution that is roughly isotropic (Fig. 8). This is expected for the large sample of stars analysed in this study and emphasises that our method is not affected by bias coming from sample selection effects. The inclination estimates obtained between 12 and $40^{\circ}$ in Fig. 6 have been redistributed towards lower inclinations, and the estimates derived between 62 and $85^{\circ}$ have been redistributed towards higher inclinations.

K19 highlighted that it is easier to identify modes when the star presents one or two rotational components compared to three components, as the power distribution between modes with different azimuthal orders leads to higher mode visibilities in these two configurations. This can lead to an observational bias when checking the inclination distribution, with a possible excess of stars with low and high inclinations compared to stars with intermediate $i$ values. Figure 8 demonstrates that our method allows us to identify triplets as efficiently as singlets and doublets, as the recovered inclination distribution is compatible with isotropy. Hence, modes are still easily identified in the case of stars presenting three rotational components, even if they have lower relative heights compared to stars with one or two components.

Our results confirm the necessity to take uncertainties on the estimated angle into account through PDFs in order to recover the underlying inclination distribution, correcting the biased distribution obtained in Fig. 6 and obtaining the reconstructed isotropic one in Fig. 8. The fact that we recover an inclination distribution compatible with an isotropic trend when randomly analysing a large number of stars validates the approach developed in this work, which can be directly applied to any solartype pulsator for which the azimuthal order of oscillation modes is identified.

\subsection{Statistical analysis}

In order to quantify the extent to which the reconstructed distribution of inclinations in Fig. 8 is compatible with isotropy,

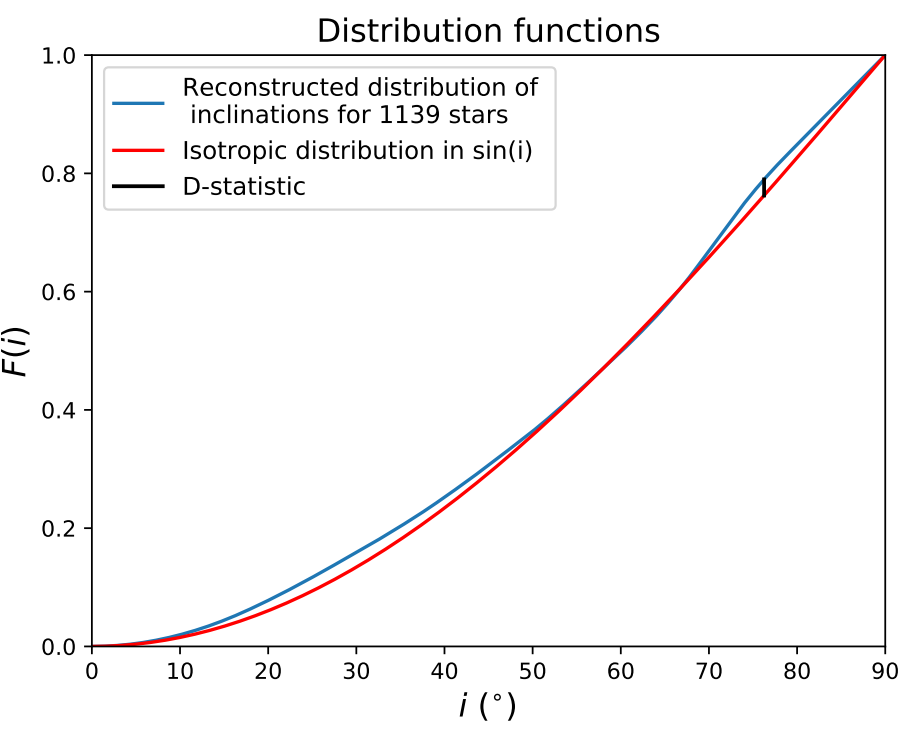

Fig. 9. Cumulative distribution functions of our sample of 1139 stars (in blue) computed from the inclination distribution in Fig. 8, and of a purely isotropic distribution in $\sin (i)$ (in red). The D-statistic is highlighted in black.

we performed a Kolmogorov-Smirnov (K-S) test (Kolmogorov 1933; Smirnov 1939) as used by K19. The K-S test consists in comparing the cumulative distribution functions (CDFs) $F_{1}(x)$ and $F_{2}(x)$ of two samples of size $n_{1}$ and $n_{2}$ by computing the maximum deviation between them at fixed inclination angle. In this work, we use a one-sample K-S test because we compare the CDF of our observational sample of size $n=1139$ with a theoretical isotropic one (Kolmogorov 1933). We computed the maximum deviation (D-statistic) between the CDF of our sample and the CDF of a purely isotropic distribution and found $D=2.72 \%$ (Fig. 9).

The $\mathrm{H}_{0}$ hypothesis we considered is that our observational sample comes from a purely isotropic distribution of inclinations 


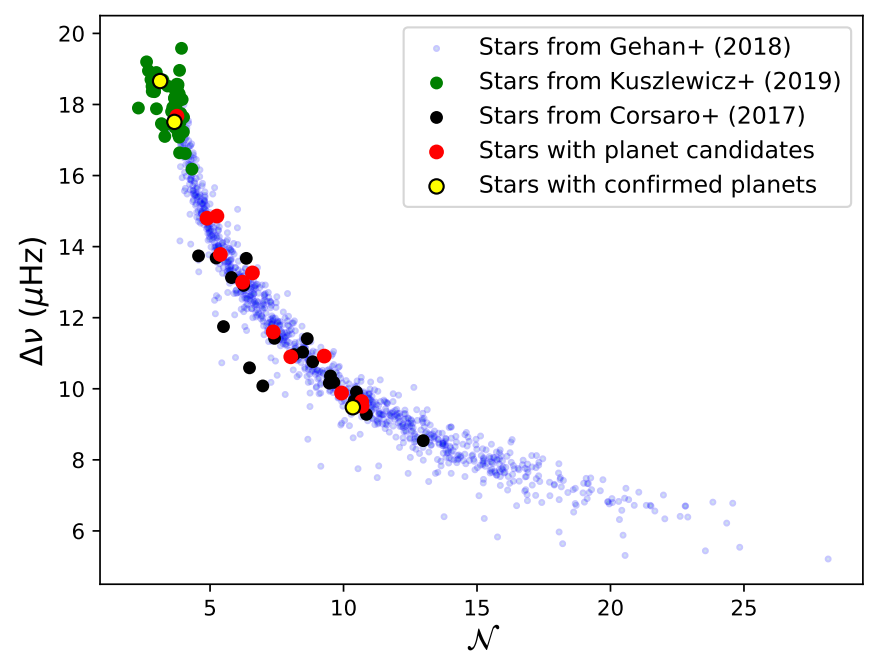

Fig. 10. Large separation as a function of the mixed-mode density. Stars analysed in Sect. 3 are in blue, stars in common with Kuszlewicz et al (2019a) are in green, stars in common with Corsaro et al. (2017) are in black, stars with planet candidate(s) are in red, and stars with confirmed planet(s) are in yellow.

(Santos et al. 2015). For samples of size $n>35$, the $\mathrm{H}_{0}$ hypothesis is rejected if (Smirnov 1939)

$D>\frac{c(\alpha)}{\sqrt{n}}=D_{\mathrm{H}_{0}}$,

where $c(\alpha)$ is a constant coming from the one-sample K-S table (Smirnov 1939) corresponding to a probability $\alpha$ of incorrectly rejecting the $\mathrm{H}_{0}$ hypothesis. We consider values of $\alpha$ from 1 to $10 \%$ to check what evidence there may be to reject $\mathrm{H}_{0}$. We note that $\alpha=10 \%$ provides only weak evidence for the rejection of the null hypothesis, and therefore there is no justification to consider values larger than that. For $\alpha=10 \%$, the K-S table gives $c(\alpha)=1.22$, from which we obtain $D_{\mathrm{H}_{0}}=4.83 \%$. This value is above $D=2.72 \%$ computed for our sample, which indicates that the $H_{0}$ hypothesis is not rejected at the $10 \%$ level, and that no significant deviation from isotropy is detected. We emphasise that the non-rejection of the $H_{0}$ hypothesis does not allow us to consider that the $H_{0}$ hypothesis is verified. However, the non-rejection of the $H_{0}$ hypothesis indicates that the distribution of inclinations derived in this study is fully compatible with isotropy, as expected.

K19 and C17 determined the inclination of some Kepler RGB and red clump stars through a Bayesian analysis of oscillation spectra. Below we explore the compatibility of these results with ours.

\section{Comparison with Kuszlewicz et al. (2019a) results}

K19 derived inclination measurements for 89 RGB stars. These latter authors selected stars with high $v_{\max }$ values, which are therefore mostly above the confusion limit between rotational splittings and mixed-mode frequency spacings (Mosser et al 2012a), for which the identification of mixed modes is straightforward without using stretched oscillation spectra (Mosser et al. 2015) as in Gehan et al. (2018). Using the mixed-mode density defined in Eq. (6) as a proxy of stellar evolution on the RGB (Gehan et al. 2018), we note that these stars lie on the lower RGB, with $\mathcal{N}$ values below 5 (in green in Fig. 10). The 1139

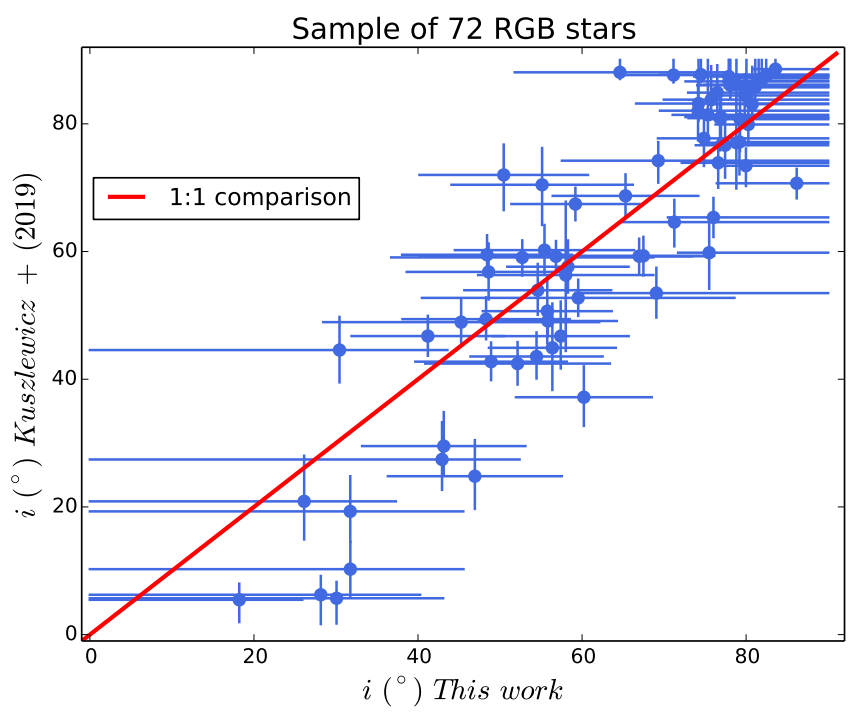

Fig. 11. Star-by-star comparison between Kuszlewicz et al. (2019a) measurements and ours. The red line represents the 1:1 comparison.

stars analysed in this study have higher $\mathcal{N}$ values on average, namely as high as 28 (blue in Fig. 10). Indeed, using stretched periods allows us to identify rotational multiplets even when they overlap, and therefore we can work at much lower $v_{\max }$ than K19 and we are not limited to the lower RGB only. We were able to derive inclination measurements for 72 stars analysed by K19 (Table A.1). We were not able to draw conclusions for 17 stars for which $g-m$ modes have low HBR.

\subsection{Raw stellar inclinations}

Our results globally agree with those of K19 for the 72 stars analysed (Fig. 11). The oscillation spectra and the stretchedperiod échelle diagrams of some of these stars are shown in Appendix B, with the azimuthal order of mixed modes identified. The small differences we find for some stars can be explained by the fact that K19 included $p-m$ modes to derive an estimate of the inclination, and not only $g-m$ modes as in our study. We note that K19 used a Bayesian hierarchical method that provides inclination measurements derived from the combination of local estimates of $i$ instead of coming from a global analysis using all modes with the same azimuthal order together, as we did in this study. While Kamiaka et al. (2018) highlighted the fact that fitting oscillation modes individually can lead to biases in the inclination measurement, K19 used synthetic oscillation spectra to verify that using hierarchical inference makes it possible to reliably extract $i$, even when using a local analysis.

We also note that we have much larger uncertainties than those derived by K19 (Fig. 11). K19 determined uncertainties as the $68.3 \%$ highest posterior density interval, which corresponds to formal 1- $\sigma$ uncertainties when the posterior distribution follows a normal law. Our uncertainties are also 1- $\sigma$, except with lower error bars for stars presenting only one rotational component and upper error bars for some stars presenting two rotational components. K19 used a Bayesian approach based on an isotropic prior for the inclination. They assumed that individual angles are all drawn from an isotropic underlying distribution, allowing the inclination angle of individual modes to differ slightly under the assumption that these differences are small. By contrast, we adopt a frequentist approach with no prior on 


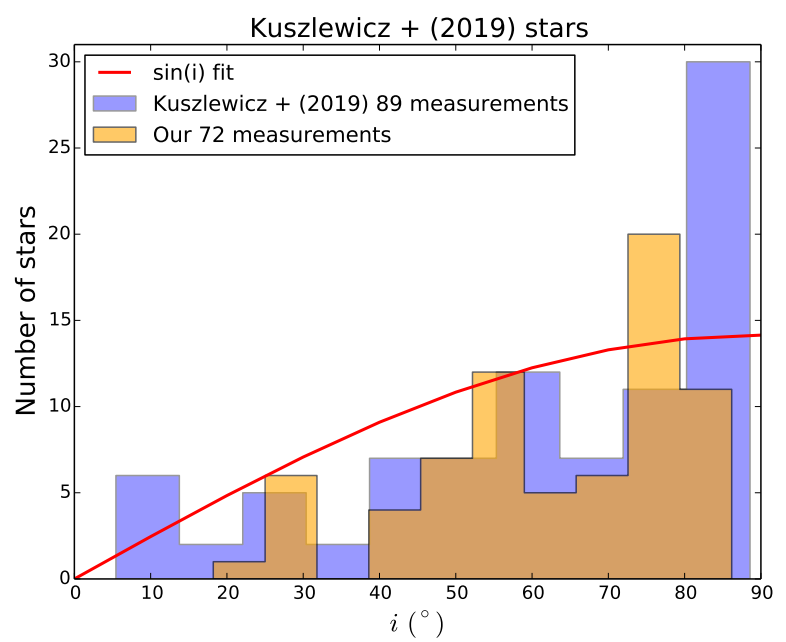

Fig. 12. Distribution of measured inclinations for the sample studied by Kuszlewicz et al. (2019a). The distribution resulting from Kuszlewicz et al. (2019a) measurements for 89 stars is in blue, and the distribution resulting from our measurements for 72 stars is in orange. The red line represents an isotropic distribution in $\sin (i)$ deriving from a fit to the histogram in blue.

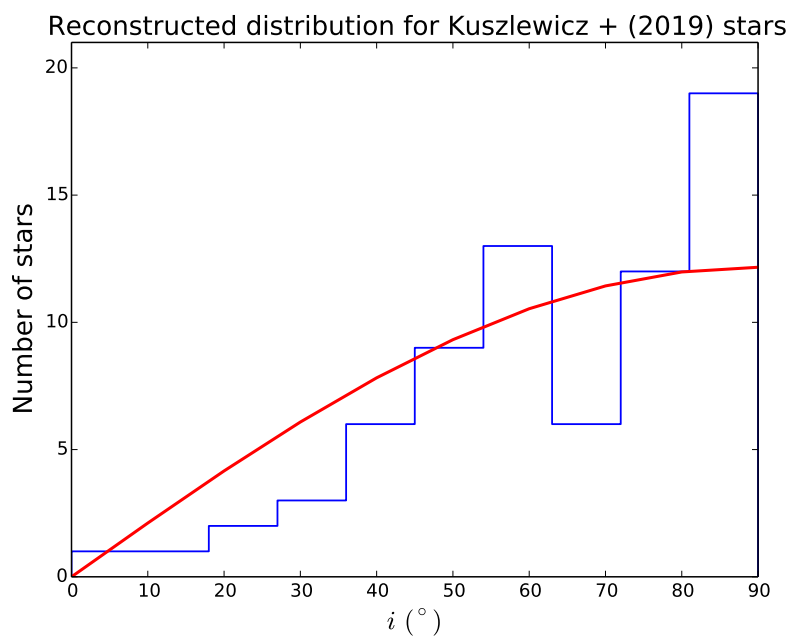

Fig. 13. Reconstructed distribution of stellar inclinations such as Fig. 8 for the 72 stars we have in common with Kuszlewicz et al. (2019a). The red line represents an isotropic distribution in $\sin (i)$ derived from a fit to the histogram.

the possible values of individual inclinations. We derive our uncertainties considering the spread in PSD for all modes with the same azimuthal order. These variations in PSD for a given azimuthal order can be significant along the spectrum due to the stochastic nature of oscillations. Therefore, it is not surprising that we end up with larger uncertainties compared to K19.

We obtain a very similar distribution of inclinations to that found by K19, the main difference being that our distribution is squeezed with no extreme low and high inclination values (Fig. 12).

\subsection{Unbiased inclination distribution}

The reconstructed histogram of inclinations using PDFs for each of the 72 stars from the K19 sample (Fig. 13) is not isotropic, in contrast to the reconstructed distribution we obtain for 1139 stars (Fig. 8). It is not too surprising that we do not recover a clean

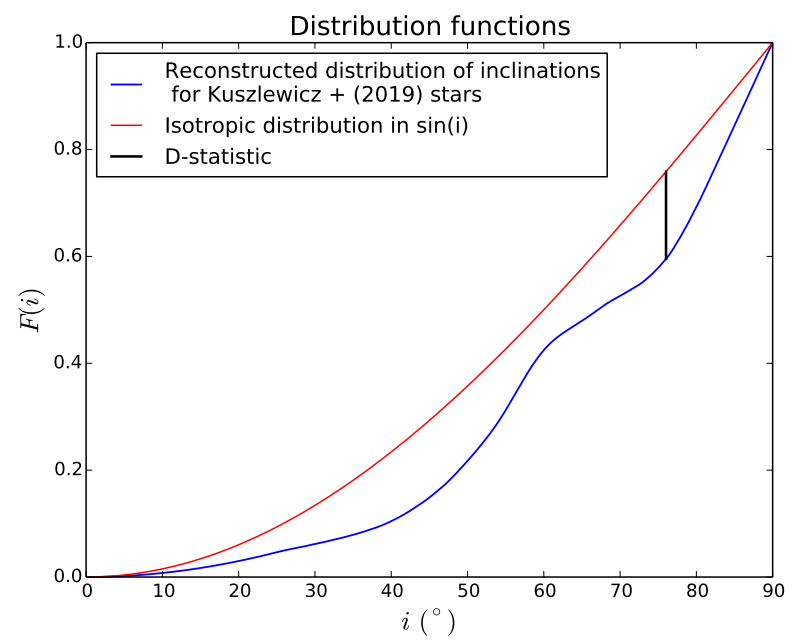

Fig. 14. Cumulative distribution functions of the sample of 72 stars from Kuszlewicz et al. (2019a) (blue) computed from the inclination distribution in Fig. 13, and of a purely isotropic distribution in $\sin (i)$ (red). The D-statistic is highlighted in black.

isotropic distribution because the sample analysed is limited. We can however note that there is a bump in the distribution around $55^{\circ}$ as well as a clear overestimate of high inclinations above $80^{\circ}$. K19 stated that the mode visibilities are higher for modes in singlets or doublets than for modes in triplets, making it easier to identify oscillation modes for these configurations. Under this assumption, the K19 sample is expected to have a deficit of intermediate inclinations. The isotropic distribution of inclinations results in a low probability of observing a star with $i$ close to $0^{\circ}$. An overestimate of stars with $i$ close to $90^{\circ}$ is therefore expected if the observational bias induced by the relative heights of oscillation modes is not corrected. Figure 13 may suggest that K19 could not overcome this bias, which could potentially result in a non-isotropic sample of stars for which they derive the inclination angle.

\subsection{Statistical analysis}

In order to quantify the extent to which the reconstructed distribution of inclinations in Fig. 13 is representative of a sample of 72 stars selected among an isotropic inclination distribution, we performed a K-S test in the same way as in Sect. 3.3.

We computed the D-statistic for the CDF of our sample of 72 stars with respect to the CDF of a purely isotropic distribution and found $D=16.33 \%$ (Fig. 14). For the rejection level $\alpha=5$ $\%$, we have $c(\alpha)=1.36$ from the K-S table and we obtain $D_{\mathrm{H}_{0}}=$ $16.03 \%$ for our sample of size $n=72$ (Eq. (20)). This value is below $D=16.33 \%$ computed for our sample. This indicates that the $H_{0}$ hypothesis is rejected at the $5 \%$ level, and we can state that the inclination distribution we have is not isotropic.

These results from the K-S test are consistent with the analysis of $\mathrm{K} 19$, who found $D \simeq 17 \%$ for the 89 stars they analysed (Fig. 8 of K19). The corresponding D-statistic necessary to reject the $H_{0}$ hypothesis at the $2 \%$ level is $D_{\mathrm{H}_{0}}=16.11 \%$ for a sample of size $n=89$ (Eq. (20)). The $H_{0}$ hypothesis is therefore also rejected at the $2 \%$ level for the total sample analysed by K19.

This statistical analysis emphasises the fact that K19 could only derive inclinations for a biased sample, which is intrinsically non-isotropic. This is not surprising because they were able to fit 89 stars out of their sample of 123 stars. This bias comes from the relative mode visibilities that lead to an easier 
C. Gehan et al:: Automated approach to measure stellar inclinations: validation on the red giant branch

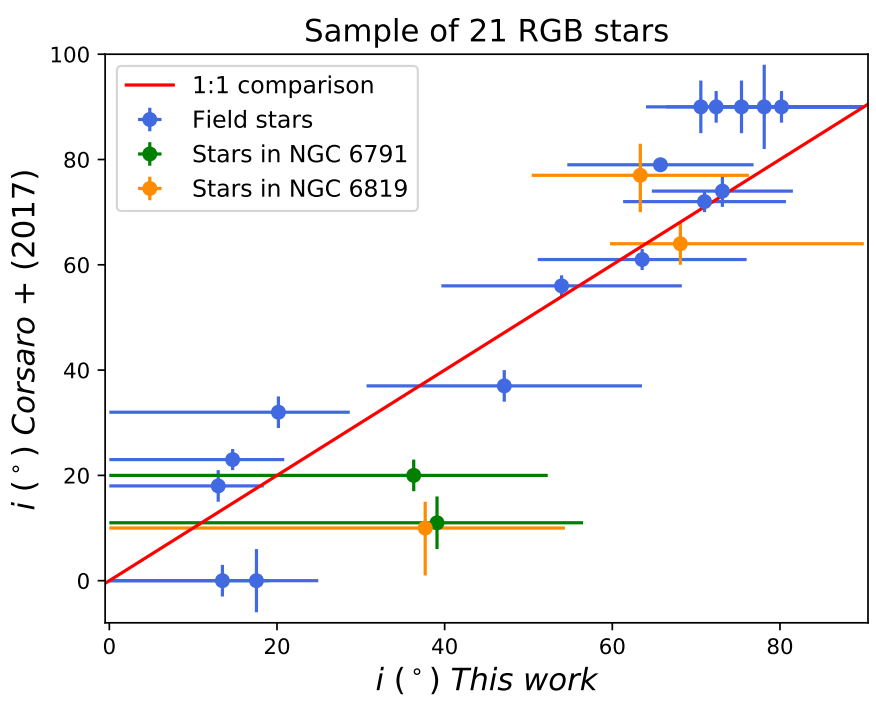

Fig. 15. Star-by-star comparison between Corsaro et al. (2017) measurements and ours for RGB stars. Field stars are in blue, stars from NGC 6791 are in green, and stars from NGC 6819 are in orange. The red line represents the 1:1 comparison.

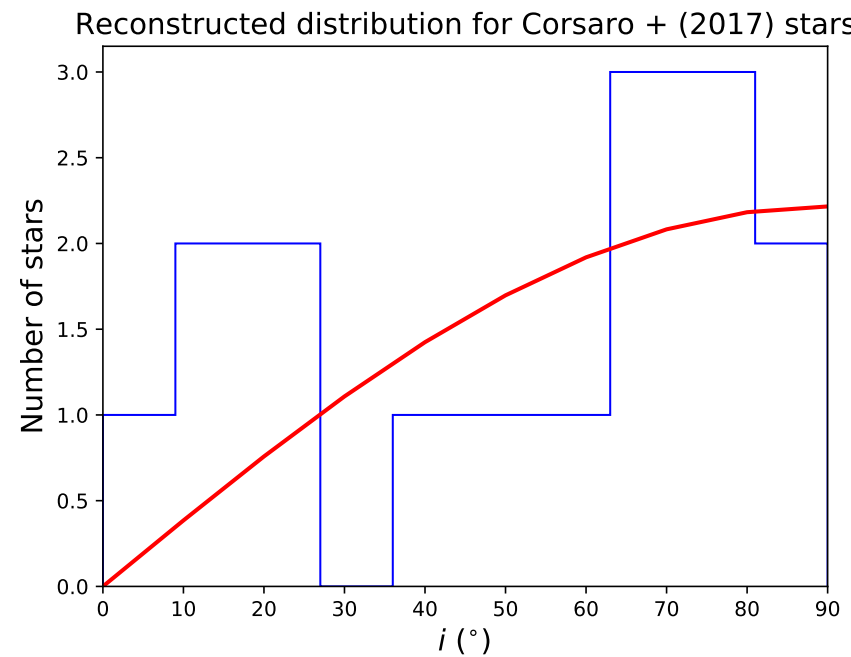

Fig. 16. Reconstructed distribution of stellar inclinations such as Fig. 8 for the 16 RGB stars we have in common with Corsaro et al. (2017). The red line represents an isotropic distribution in $\sin (i)$ derived from a fit to the histogram.

identification of mixed modes when the inclination is close to $55^{\circ}$ or close to $90^{\circ}$. This evidence does not diminish the interest of their work.

\section{Comparison with Corsaro et al. (2017) results}

C17 derived inclination measurements for 84 RGB and clump stars. Of these, 36 are field stars, 25 belong to the open cluster NGC 6791, and 23 to the open cluster NGC 6819. These latter authors find that low inclinations are favoured in the two open clusters NGC 6791 and NGC 6819, and therefore that inclinations do not follow the expected isotropic distribution. They concluded that stellar spins seem to be preferentially aligned towards the line of sight for those two open clusters. This is a relatively unexpected result that was contradicted by Mosser et al. (2018), who reanalysed C17 stars belonging to NGC 6819 and

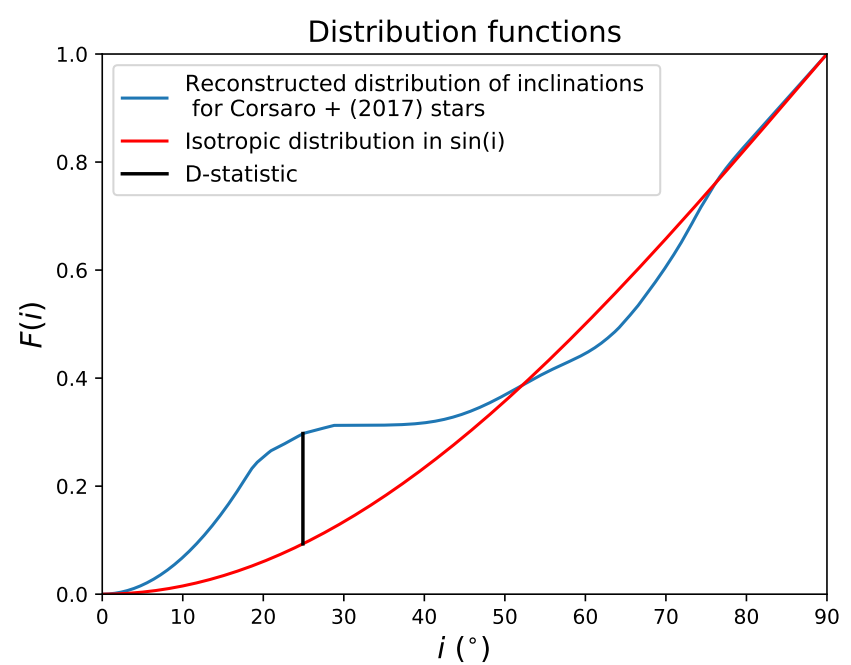

Fig. 17. Cumulative distribution functions of the sample of 16 RGB field stars from Corsaro et al. (2017) (blue) computed from the inclination distribution in Fig. 16, and of a purely isotropic distribution in $\sin (i)$ (red). The D-statistic is highlighted in black.

found that the distribution of inclinations follows an isotropic trend.

The method of Gehan et al. (2018) is not suitable for analysing clump stars. Indeed, oscillation modes present larger mode line widths combined with lower rotational splitting values compared to RGB stars, making rotational components very hard to disentangle because they are very close to each other in stretched-period échelle diagrams (extension of the upper part of Fig. 3 of Gehan et al. 2018). We therefore focus here on the comparison between our results and those of C17 for RGB stars only. C17 have 36 RGB stars in their sample, including 19 field stars, 13 stars belonging to NGC 6791, and 4 stars belonging to NGC 6819. We were able to derive inclination measurements for 21 RGB stars analysed by C17, including 16 field stars, 3 stars from NGC 6819, and 2 stars from NGC 6791 (Table A.2 and figures in Appendix B). We were not able to form conclusions for all stars analysed by $\mathrm{C} 17$ because some of them have a low HBR for $g-m$ modes, in particular for the open cluster NGC 6791.

\subsection{Raw stellar inclinations}

The measurements we derived for RGB stars are in global agreement with those obtained by $\mathrm{C} 17$, not only for field stars but also for cluster stars (Fig. 15). As we already noted for K19 results, uncertainties derived by $\mathrm{C} 17$ are much smaller than ours, while corresponding to a $1-\sigma$ confidence interval. These differences are likely due to the different approaches used: $\mathrm{C} 17$ used a Bayesian approach including a prior on the inclination, while we used a frequentist approach with no prior to derive our measurements. Additionally, C17 were able to measure inclinations as low as $0^{\circ}$ for two RGB stars that we analysed and as high as $90^{\circ}$ for five stars (Fig. 15), which is not possible as explained in in Sect. 2.3.2.

\subsection{Statistical analysis}

C17 claimed that the distribution of inclinations they derive for their sample of 36 RGB and clump field stars is in agreement with an isotropic distribution. We obtained the reconstructed histogram of inclinations using PDFs for each of the 16 field stars from the $\mathrm{C} 17$ sample (Fig. 16). In order to assess the extent to 


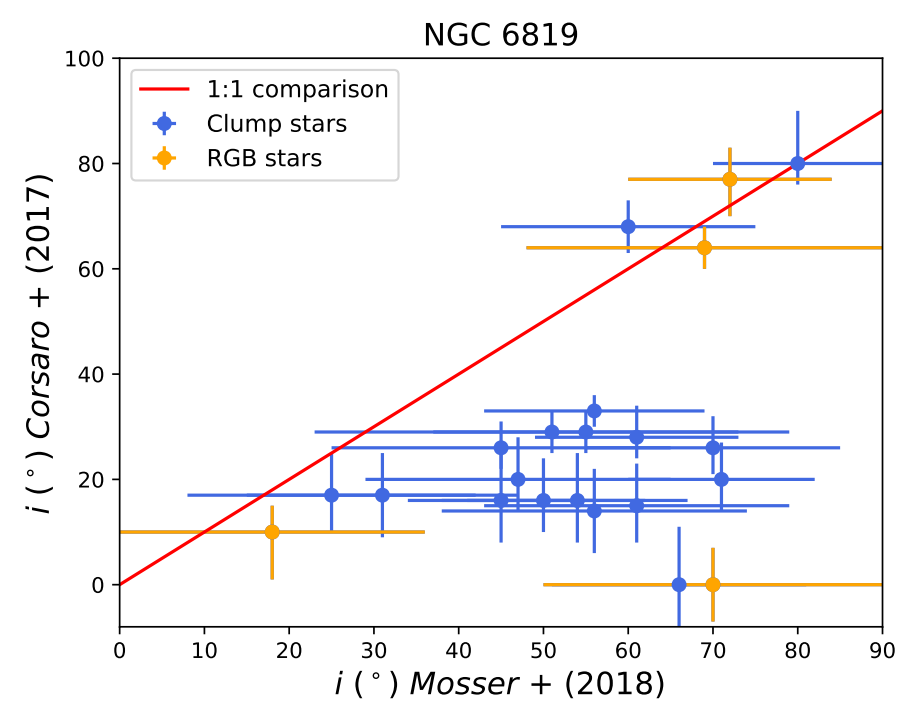

Fig. 18. Star-by-star comparison between Corsaro et al. (2017) and Mosser et al. (2018) measurements for stars belonging to NGC 6819. Clump stars are in blue, and RGB stars are in orange. The red line represents the 1:1 comparison.

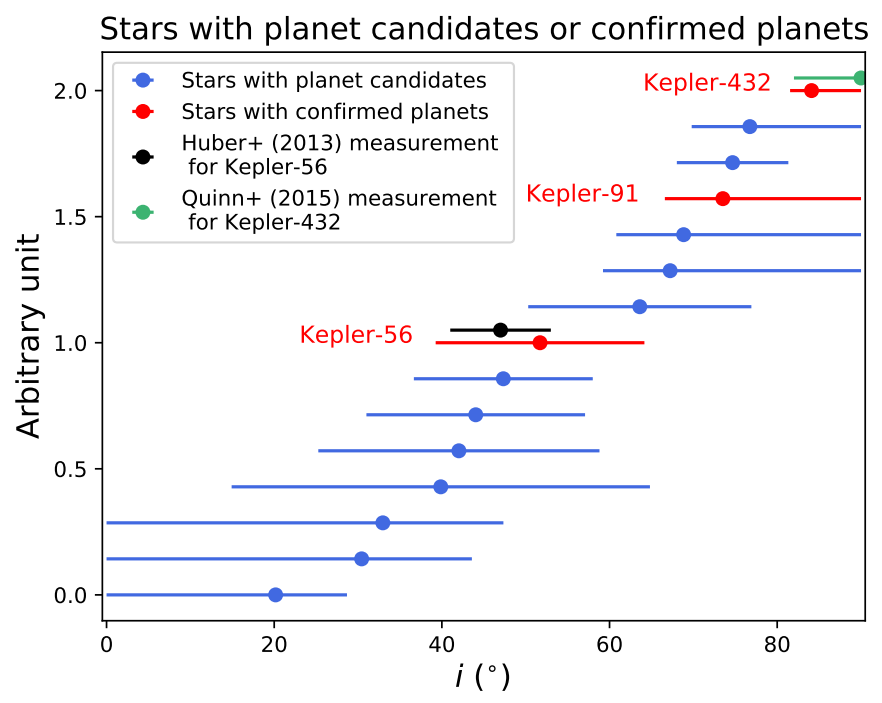

Fig. 19. Measured inclinations for stars with a planet candidate(s), in blue, and with a confirmed planet(s), in red. The $y$-axis is arbitrary. The Huber et al. (2013b) measurement for Kepler-56 with misaligned planets is shown in black, with the Quinn et al. (2015) measurement for Kepler-432 in green.

which the reconstructed distribution of inclinations is representative of a sample of 16 stars selected among an isotropic inclination distribution, we performed a K-S test in the same way as in Sects. 3.3 and 4.3.

We computed the D-statistic for the CDF of our sample of 16 field stars with respect to the CDF of a purely isotropic distribution and found $D=20.42 \%$ (Fig. 17). For our sample of size $n=16$, the K-S table directly gives (Smirnov 1939) $D_{\mathrm{H}_{0}}=29.47 \%$ for the low rejection level $\alpha=10 \%$, which is above $D=20.42 \%$ computed for our sample. This indicates that the $H_{0}$ hypothesis is not rejected to the $10 \%$ level. The inclination distribution for $\mathrm{C} 17$ stars therefore does not exhibit significant deviation from isotropy. Therefore, there is no obvious observational bias in the sample selection, as stated by $\mathrm{C} 17$.
Table 1. Properties of RGB stars with a planet candidate(s).

\begin{tabular}{lccc}
\hline \hline $\mathrm{KIC}$ & $i\left(^{\circ}\right)$ & $\sigma_{i,+}\left(^{\circ}\right)$ & $\sigma_{i,-}\left(^{\circ}\right)$ \\
\hline 4953262 & 39.9 & 25.0 & 25.0 \\
5110453 & 42.0 & 16.8 & 16.8 \\
5115688 & 30.4 & 13.2 & 30.4 \\
6425377 & 44.0 & 13.0 & 13.0 \\
7198587 & 68.8 & 21.2 & 8.0 \\
7458743 & 76.7 & 13.3 & 6.9 \\
8016650 & 33.0 & 14.4 & 33.0 \\
9145861 & 74.7 & 6.7 & 6.7 \\
9475697 & 20.2 & 8.5 & 20.2 \\
10790401 & 67.2 & 22.8 & 8.0 \\
11097752 & 63.6 & 13.3 & 13.3 \\
11911929 & 47.33 & 10.7 & 10.7 \\
\hline
\end{tabular}

\subsection{Inclinations for open clusters}

For the RGB stars analysed here, we obtain inclination measurements in agreement with those derived by C17 (Fig. 15). However, Mosser et al. (2018) obtained inclination measurements for red giants of the open cluster NGC 6819 that contradict the results of $\mathrm{C} 17$ (Fig. 18). Part of the explanation might come from the fact that the great majority of these NGC 6819 stars are clump stars, for which $\mathrm{C} 17$ overestimate low inclinations (Fig. 18). For three RGB stars out of four, results obtained by $\mathrm{C} 17$ and Mosser et al. (2018) are in agreement. Figure 18 therefore suggests that mixed modes are not always correctly identified by $\mathrm{C} 17$ in the case of clump stars. This is highlighted by Supplementary Fig. 1 of $\mathrm{C} 17$ where only $m=0$ modes are identified, resulting in an inclination of $20_{-6}^{+8^{\circ}}$, while Fig. 16 of Mosser et al. (2018) reveals that the three components with $m=\{-1,0,1\}$ are visible and the corresponding inclination is $47 \pm 18^{\circ}$. This mode misidentification is not surprising as clump stars present larger line widths together with smaller rotational splittings compared to RGB stars. In these conditions, it is more probable to identify only one rotational component while two or three are actually visible, resulting in an underestimated inclination. The conclusion of $\mathrm{C} 17$ regarding aligned stellar spins in clusters must therefore be considered with caution. As our method is not suitable for clump stars, we cannot directly check this hypothesis by deriving $i$ measurements for clump stars in the open clusters NGC 6819 and 6791. This merits a dedicated study with another method to analyse clump stars.

\section{Red giants with a planet candidate(s) or a confirmed planet(s)}

As mentioned in Sect. 1, inclination measurements for planethosting stars provide information on the obliquity of planetary systems (Eq. (1)). For stars with transiting planet(s), we expect that, on average, the lower the inclination, the larger the obliquity, and therefore the higher the degree of spin-orbit misalignment between the host star and the planet(s). In this context, we used the NASA Exoplanet Archive cumulative table listing Kepler Objects of Interest (KOI) ${ }^{2}$ to lead a systematic search for RGB stars in our sample with a confirmed planet(s) and for stars with a planet candidate(s) that have not yet been confirmed or dismissed.

\footnotetext{
2 https://exoplanetarchive.ipac.caltech.edu/cgi-bin/
} TblView/nph-tblView?app=ExoTbls\&config=cumulative 
Table 2. Properties of RGB stars with a confirmed planet(s).

\begin{tabular}{lcccccc}
\hline \hline Name & KIC & Number of planets & $\mathcal{N}$ & $i\left(^{\circ}\right)$ & $\sigma_{i,+}\left(^{\circ}\right)$ & $\sigma_{i,-}\left({ }^{\circ}\right)$ \\
\hline Kepler-56 & 6448890 & 3 & 3.7 & 51.7 & 12.5 & 12.5 \\
Kepler-91 & 8219268 & 1 & 10.4 & 73.5 & 16.5 & 6.9 \\
Kepler-432 & 10864656 & 2 & 3.1 & 84.1 & 5.9 & 2.6 \\
\hline
\end{tabular}

\subsection{Inclination and evolutionary stage}

Among all the stars analysed in this study, we found 12 RGB stars in our sample with a planet candidate(s). These stars have inclinations distributed in the $\left[0,90^{\circ}\right]$ range, with five stars presenting intermediate inclinations and three stars presenting low inclinations, being possibly pole-on (Fig. 19 and Table 1). We suggest that these 12 RGB stars should be analysed in priority to assess whether or not they have a confirmed transiting planet(s). If these stars turn out to host a planet(s), it will be possible to put constraints on the obliquity of the planetary orbit because we have measured their inclination angle. The eight aforementioned stars with low and intermediate inclinations should in particular present large obliquities should a transiting planet(s) be confirmed. It is interesting to note that these stars present diverse evolutionary stages on the RGB, with mixedmode density values between $\mathcal{N}=3.8$ and 10.7 (Fig. 10). Any planet detection around these stars would provide constraints on planetary evolution and survival around evolved stars, until relatively far up on the RGB where stars already started to expand significantly. We also find one RGB star in our sample belonging to an eclipsing binary system, KIC 8564976, which was also known as KOI-3890 before its binary nature was confirmed by Kuszlewicz et al. (2019b). We find an inclination of $i=74.6_{-7.7}^{+15.4}$ 。 for this star, consistent with the value of $i=87.3_{-1.1}^{+2.7}$ found by Kuszlewicz et al. (2019b).

Additionally, we find no stars with confirmed planet(s) among stars analysed in this study. We therefore considered the seven Kepler evolved stars hosting exoplanet(s) presented by Lillo-Box et al. (2016) and were able to measure the inclination of three of these stars (Fig. 19 and Table 2). Two stars have large inclination values consistent with a star seen almost equator-on, and therefore the planetary orbit axis is likely to be approximately aligned with the star's rotation axis. Our measurement of $i=84.1_{-2.6}^{+5.9 \circ}$ for Kepler-432 is compatible with Quinn et al. (2015), who measured $i=90_{-8}^{+0 \circ}$ (Fig. 19). We measured an inclination of $i=51.7 \pm 12.5^{\circ}$ for Kepler-56, which is consistent with Huber et al. (2013b) who measured $i=47 \pm 7^{\circ}$ (Fig. 19), from which they deduced a lower limit for the obliquity of $\psi>37^{\circ}$. Unsurprisingly, two of these three host stars are on the very low RGB, with mixed-mode density values of $\mathcal{N}=3.1$ and 3.7 respectively (Fig. 10 and Table 2). However, the third star (Kepler-91) is more evolved, with $\mathcal{N}=10.4$ (Fig. 10 and Table 2). Seismic scaling relations give $R \sim 7.33 R_{\odot}$ for this star, which is compatible with $R=6.30 \pm 0.16 R_{\odot}$ indicated in Lillo-Box et al. (2016). This confirms that planets can survive far up on the RGB and not only on the early RGB phase.

\subsection{Planetary orbit angle for a transiting planet}

One might be tempted to consider that $i_{\mathrm{p}} \simeq 90^{\circ}$ for transiting planets, and therefore that Eq. (1), which defines the obliquity, can be simplified to (Kamiaka et al. 2018)

$\cos \psi \simeq \sin i \cos \lambda$.

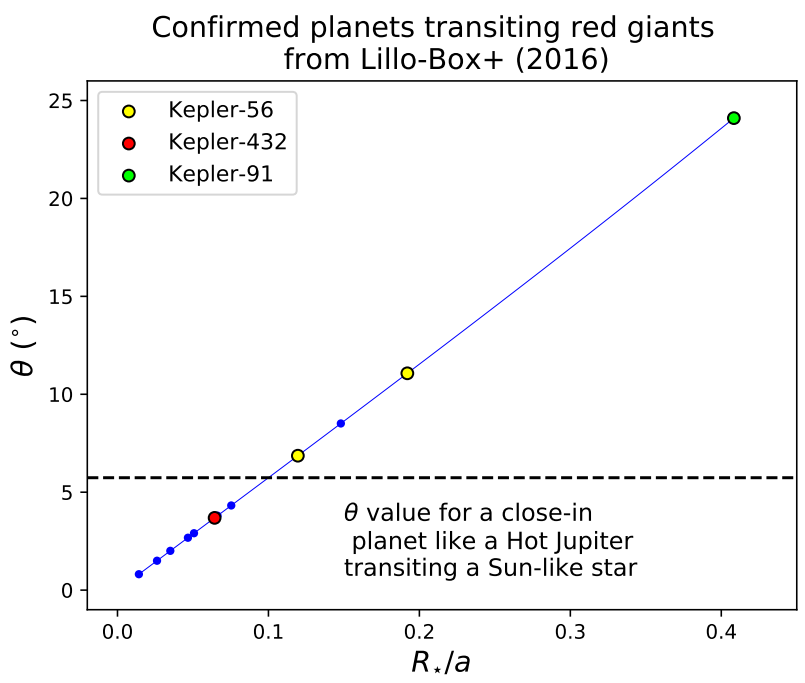

Fig. 20. Deviation angle $\theta$ (Eq. (23)) as a function of the ratio between the stellar radius and the planetary semi-major axis for Kepler RGB stars with confirmed transiting planets listed in Lillo-Box et al. (2016). Planets around RGB stars analysed in this study are coloured according to their host star. The black dashed line indicates the maximum $\theta$ value for planets transiting Sun-like stars computed for close-in planets like hot Jupiters, $\theta \simeq 5.7^{\circ}$.

However, a transit can occur if the inclination of the planetary orbit follows

$\left(90^{\circ}-\theta\right) \leq i_{\mathrm{p}} \leq\left(90^{\circ}+\theta\right)$,

with, assuming that the planet radius is much smaller than the stellar radius,

$\theta=\arcsin \left(\frac{R_{\star}}{a}\right)$,

where $R_{\star}$ is the stellar radius and $a$ is the semi-major axis of the planet (Beatty \& Seager 2010).

When considering transiting planets around Sun-like stars, Eq. (21) is mostly valid, with $\theta$ taking maximum values around $5.7^{\circ}$ for hot Jupiters that have typically $R_{\star} / a=1 / 10$ (Beatty \& Seager 2010). We nevertheless note that, although we can still safely consider that $\sin i_{\mathrm{p}} \simeq 1$ in the left-hand term of Eq. (1) for $\theta$ values as high as $5.7^{\circ}$, we have $\cos i_{\mathrm{p}} \simeq 0.1$ for $\theta=5.7^{\circ}$. We therefore advocate caution when considering that $\cos i_{\mathrm{p}} \simeq 0$ in the right-hand term of Eq. (1) for close-in planets transiting Sun-like stars, such as hot Jupiters.

Nevertheless, Eq. (21) can be inappropriate in many cases for transiting planets around evolved stars with larger radii. We considered planets around red giants listed by Lillo-Box et al. (2016) and used the Extrasolar Planet Encyclopedia ${ }^{3}$ to keep those that were detected by the transit method. All Kepler planets in Lillo-Box et al. (2016) are transiting planets, except

3 http://exoplanet.eu/catalog/all_fields/ 
Kepler-56d and Kepler-432c. Of the 12 transiting planets from Lillo-Box et al. (2016), 4 have $\theta$ values above the maximum value of $\theta=5.7^{\circ}$ for Sun-like stars, including 3 planets that are transiting RGB stars analysed in this study (Fig. 20). The planet around the most evolved RGB star (Kepler-91), which also has the largest radius, has the most important $\theta$ value with $\theta=24.1^{\circ}$. As a consequence, while one can safely apply Eq. (21) to all planets around Sun-like stars up to close-in planets like hot Jupiters, this is no longer systematically true for more evolved stars with larger radii, and one has to consider Eq. (1) as a whole in many cases.

\section{Conclusion}

This work highlights the biases that affect stellar inclination measurements and provides a way to infer the underlying statistical distribution of inclinations for a given population of stars.

We developed a general and automated approach to derive seismic measurements of the stellar inclination that can be applied to any solar-type pulsator for which oscillation modes are identified. We analysed RGB stars observed by Kepler, including mainly field stars. Raw inclinations that are measured are not isotropically distributed, in contrast to what is expected for random inclinations. In particular, values of $i \lesssim 10^{\circ}$ and $i \gtrsim 85^{\circ}$ cannot be directly measured, and this observational limitation results in a biased distribution. The expected isotropic distribution is actually recovered when taking into account individual uncertainties on the inclination measurement for each star using PDFs, validating the method setup in this study. Our results emphasise the fact that unveiling the statistical significance of the inclination distribution of a given sample of stars is difficult, and that considering uncertainties in an accurate way is fundamental to retrieving the true underlying distribution.

Additionally, we were able to confirm that the distribution of inclinations derived by Kuszlewicz et al. (2019a) for stars on the lower RGB is not isotropic and results from a bias in sample selection. We also checked the results obtained by Corsaro et al. (2017) and derived inclination measurements that are in agreement for field stars on the RGB. For the open clusters NGC 6791 and NGC 6819, we were only able to analyse a handful of RGB stars. Results obtained by Mosser et al. (2018) for red giants in NGC 6819 suggest that mixed-mode misidentification is more common for clump stars analysed by Corsaro et al. (2017) than for RGB stars, calling into question the stellar spin alignment inferred by Corsaro et al. (2017) for the two open clusters NGC 6791 and NGC 6819. The analysis of clump stars is beyond the scope of this paper and merits a dedicated study to check this assumption in detail.

Moreover, we measured the inclination angle of 12 RGB stars with a planet candidate or candidates. These should be analysed in priority to assess whether or not they host planets as it would be possible to put constraints on the obliquity of the planetary orbit, with eight systems that should present large obliquities. We also derived the inclination of three RGB stars with a confirmed planet or planets, and our results are in agreement with previous measurements. Kepler-91 and Kepler432 have large inclination values consistent with a low obliquity, and we confirm that the multiplanetary system Kepler-56 has an intermediate inclination angle, for which Huber et al. (2013a) inferred a high obliquity corresponding to misaligned planets. We find that Kepler-56 and Kepler-432 unsurprisingly lie on the lower RGB. However, Kepler-91 is more evolved and has a larger radius, confirming that planets can survive relatively far up on the RGB.
Finally, we show that considering that the inclination angle of the planetary orbit can be safely approximated to $90^{\circ}$ in the case of transiting planets is not always valid when the host star is a red giant with a larger radius than that of main sequence stars, which greatly impacts the determination of the obliquity. This assumption should therefore be systematically verified before deriving an estimate of the obliquity for planets transiting red giants.

This work revisits the problem of inferring the distribution of inclinations in large stellar samples. Such studies applied to open clusters can provide valuable insight into the physical conditions driving star formation. Kepler observed only four open clusters with large distance moduli (Bossini et al. 2019). The undergoing TESS space mission (Aguirre et al. 2020) has a near full-sky coverage, including a lower limit of about 600 open clusters (Bouma et al. 2019). We can therefore expect to have a significant number of open clusters observed by TESS with smaller distance moduli, potentially presenting higher signalto-noise ratios compared to Kepler clusters. As TESS has now two years of observations and the extended mission is now approved, we can expect a sufficiently high resolution to disentangle individual mixed modes in the oscillation spectra of red giants belonging to open clusters. This would allow us to unveil the physical conditions during star formation for tens and potentially hundreds of open clusters.

Acknowledgements. C. G. thanks Tiago Campante for helpful discussions as well as D. Stello, J. S. Kuszlewicz and K. J. Bell for constructive comments. This work was supported by FCT - Fundacao para a Ciência e a Tecnologia through national funds (PTDC/FIS-AST/30389/2017), by FEDER - Fundo Europeu de Desenvolvimento Regional through COMPETE2020 - Programa Operacional Competitividade e Internacionalizacao (POCI-01-0145-FEDER030389), and by FCT/MCTES through national funds (PIDDAC) by these grants UIDB/04434/2020 and UIDP/04434/2020. M. S. C. is supported by FCT through a contract (CEECIND/02619/2017).

\section{References}

Aguirre, V. S., Stello, D., Stokholm, A., et al. 2020, ApJ, 889, L34 Albrecht, S., Winn, J. N., Johnson, J. A., et al. 2012, ApJ, 757, 18 Albrecht, S., Winn, J. N., Marcy, G. W., et al. 2013, ApJ, 771, 11 Appourchaux, T., Michel, E., Auvergne, M., et al. 2008, A\&A, 488, 705 Beatty, T. G., \& Seager, S. 2010, ApJ, 712, 1433

Beck, P. G., Montalban, J., Kallinger, T., et al. 2012, Nature, 481, 55 Benomar, O., Bedding, T. R., Mosser, B., et al. 2013, ApJ, 767, 158 Bossini, D., Vallenari, A., Bragaglia, A., et al. 2019, A\&A, 623, A108 Bouma, L. G., Hartman, J. D., Bhatti, W., Winn, J. N., \& Bakos, G. Á. 2019, ApJS, 245, 13

Campante, T. L., Lund, M. N., Kuszlewicz, J. S., et al. 2016, ApJ, 819, 85 Chaplin, W. J., Sanchis-Ojeda, R., Campante, T. L., et al. 2013, ApJ, 766, 101 Chatterjee, S., Ford, E. B., Matsumura, S., \& Rasio, F. A. 2008, ApJ, 686, 580 Corsaro, E., Lee, Y.-N., García, R. A., et al. 2017, Nat. Astron., 1, 0064

Cunha, M. S., Avelino, P. P., Christensen-Dalsgaard, J., et al. 2019, MNRAS, 490, 909

Deheuvels, S., García, R. A., Chaplin, W. J., et al. 2012, ApJ, 756, 19

Deheuvels, S., Doğan, G., Goupil, M. J., et al. 2014, A\&A, 564, A27

Deheuvels, S., Ballot, J., Beck, P. G., et al. 2015, A\&A, 580, A96

Deheuvels, S., Ouazzani, R. M., \& Basu, S. 2017, A\&A, 605, A75

Fabrycky, D., \& Tremaine, S. 2007, ApJ, 669, 1298

Fabrycky, D. C., \& Winn, J. N. 2009, ApJ, 696, 1230

Gehan, C., Mosser, B., Michel, E., Samadi, R., \& Kallinger, T. 2018, A\&A, 616, A 24

Gilliland, R. L., Brown, T. M., Christensen-Dalsgaard, J., et al. 2010, PASP, 122, 131

Gizon, L., \& Solanki, S. K. 2003, ApJ, 589, 1009

Goupil, M. J., Mosser, B., Marques, J. P., et al. 2013, A\&A, 549, A75

Hekker, S., \& Christensen-Dalsgaard, J. 2017, A\&ARv, 25, 1

Heller, R. 2019, A\&A, 623, A137

Hirano, T., Masuda, K., Sato, B., et al. 2015, ApJ, 799, 9

Holt, J. R. 1893, A\&A, 12, 646 (formerly The Sidereal Messenger)

Hrudková, M., Hatzes, A., Karjalainen, R., et al. 2017, MNRAS, 464, 1018 
C. Gehan et al.: Automated approach to measure stellar inclinations: validation on the red giant branch

Huber, D. 2018, Asteroseismology and Exoplanets: Listening to the Stars and 890 Searching for NewWorlds, eds. T. L. Campante, N. C. Santos, \& M. J. P. F. G. Monteiro, 49, 119

Huber, D., Chaplin, W. J., Christensen-Dalsgaard, J., et al. 2013a, ApJ, 767, 127

Huber, D., Carter, J. A., Barbieri, M., et al. 2013b, Science, 342, 331

Johnson, J. A., Winn, J. N., Albrecht, S., et al. 2009, PASP, 121, 1104

Johnson, J. A., Bowler, B. P., Howard, A. W., et al. 2010, ApJ, 721, L153

Johnson, J. A., Clanton, C., Howard, A. W., et al. 2011, ApJS, 197, 26

Jones, M. I., Jenkins, J. S., Rojo, P., Melo, C. H. F., \& Bluhm, P. 2015, A\&A, 573, A3

Kamiaka, S., Benomar, O., \& Suto, Y. 2018, MNRAS, 479, 391

Kolmogorov, A. N. 1933, Giornale dell-Instituto Italiano degli Attuari, 4, 89

Kuszlewicz, J. S., Chaplin, W. J., North, T. S. H., et al. 2019a, MNRAS, 488, 572

Kuszlewicz, J. S., North, T. S. H., Chaplin, W. J., et al. 2019b, MNRAS, 487, 14

Lee, B. C., Park, M. G., Lee, S. M., et al. 2015, A\&A, 584, A79

Lillo-Box, J., Barrado, D., Moya, A., et al. 2014, A\&A, 562, A109

Lillo-Box, J., Barrado, D., \& Correia, A. C. M. 2016, A\&A, 589, A124

Lin, D. N. C., Bodenheimer, P., \& Richardson, D. C. 1996, Nature, 380, 606

McKee, C. F., \& Ostriker, E. C. 2007, ARA\&A, 45, 565

McLaughlin, D.B. 1924, ApJ, 60, 22

Montalbán, J., Miglio, A., Noels, A., et al. 2013, ApJ, 766, 118
Mosser, B., Belkacem, K., Goupil, M. J., et al. 2011, A\&A, 525, L9

Mosser, B., Goupil, M. J., Belkacem, K., et al. 2012a, A\&A, 548, A10

Mosser, B., Elsworth, Y., Hekker, S., et al. 2012b, A\&A, 537, A30

Mosser, B., Michel, E., Belkacem, K., et al. 2013, A\&A, 550, A126

Mosser, B., Vrard, M., Belkacem, K., et al. 2015, A\&A, 584, A50

Mosser, B., Belkacem, K., Pinçon, C., et al. 2017a, A\&A, 598, A62

Mosser, B., Pinçon, C., Belkacem, K., Takata, M., \& Vrard, M. 2017b, A\&A, 600, A1

Mosser, B., Gehan, C., Belkacem, K., et al. 2018, A\&A, 618, A109

Ohta, Y., Taruya, A., \& Suto, Y. 2005, ApJ, 622, 1118

Pinçon, C., Takata, M., \& Mosser, B. 2019, A\&A, 626, A125

Pinçon, C., Goupil, M. J., \& Belkacem, K. 2020, A\&A, 634, A68

Pinsonneault, M. H., Elsworth, Y. P., Tayar, J., et al. 2018, ApJS, 239, 32

Queloz, D., Eggenberger, A., Mayor, M., et al. 2000, A\&A, 359, L13

Quinn, S. N., White, T. R., Latham, D. W., et al. 2015, ApJ, 803, 49

Rey-Raposo, R., \& Read, J. I. 2018, MNRAS, 481, L16

Rossiter, R.A. 1924, ApJ, 60, 15

Santos, A. R. G., Cunha, M. S., Avelino, P. P., \& Campante, T. L. 2015, A\&A, 580, A62

Scuflaire, R. 1974, A\&A, 36, 107

Smirnov, N. V. 1939, Bull. Mosc. Univ., 2, 3

Vrard, M., Mosser, B., Samadi, R., et al. 2016, A\&A, 588, A87

Winn, J. N., \& Fabrycky, D. C. 2015, ARA\&A, 53, 409

Winn, J. N., Fabrycky, D., Albrecht, S., \& Johnson, J. A. 2010, ApJ, 718, L145 
Appendix A: Inclination angles derived for Kuszlewicz et al. (2019a) and Corsaro et al. (2017) stars

We provide the inclination angle, the associated uncertainties, and the number of detected rotational components we derived for the 72 Kuszlewicz et al. (2019a) stars in Table A.1 and for the 21 Corsaro et al. (2017) RGB stars in Table A.2.

Table A.1. Properties of the 72 RGB stars from Kuszlewicz et al. (2019a) sample.

\begin{tabular}{|c|c|c|c|c|}
\hline KIC & $\begin{array}{c}\text { Number of } \\
\text { rotational } \\
\text { components }\end{array}$ & $i\left(^{\circ}\right)$ & $\sigma_{i,+}\left(^{\circ}\right)$ & $\sigma_{i,-}\left({ }^{\circ}\right)$ \\
\hline 2166709 & 3 & 58.0 & 10.7 & 10.7 \\
\hline 3111383 & 1 & 31.7 & 13.8 & 31.7 \\
\hline 3446775 & 1 & 31.8 & 13.8 & 31.8 \\
\hline 3531478 & 3 & 41.2 & 9.3 & 9.3 \\
\hline 3634488 & 2 & 86.2 & 3.8 & 9.7 \\
\hline 4042882 & 3 & 48.6 & 10.0 & 10.0 \\
\hline 4139632 & 2 & 79.2 & 10.8 & 2.8 \\
\hline 4445966 & 2 & 81.1 & 8.9 & 3.2 \\
\hline 4459359 & 2 & 76.0 & 14.0 & 5.6 \\
\hline 4638467 & 3 & 55.1 & 11.1 & 11.1 \\
\hline 4646477 & 1 & 26.1 & 11.2 & 26.1 \\
\hline 4731138 & 2 & 79.8 & 10.2 & 3.1 \\
\hline 4738693 & 2 & 76.8 & 13.2 & 3.8 \\
\hline 4996676 & 1 & 30.1 & 13.0 & 30.1 \\
\hline 5033397 & 2 & 78.8 & 11.2 & 3.9 \\
\hline 5115688 & 1 & 30.4 & 13.2 & 30.4 \\
\hline 5119742 & 3 & 48.9 & 9.23 & 9.23 \\
\hline 5198982 & 3 & 56.4 & 7.7 & 7.7 \\
\hline 5305291 & 2 & 74.9 & 15.1 & 5.6 \\
\hline 5428405 & 2 & 78.1 & 11.9 & 4.0 \\
\hline 5553307 & 2 & 81.9 & 8.1 & 3.1 \\
\hline 5623097 & 3 & 48.4 & 10.4 & 10.4 \\
\hline 5649129 & 2 & 78.8 & 11.2 & 4.0 \\
\hline 5731852 & 2 & 74.5 & 15.5 & 5.0 \\
\hline 5773365 & 2 & 77.4 & 12.6 & 3.6 \\
\hline 5879486 & 2 & 75.3 & 14.7 & 4.3 \\
\hline 5961985 & 2 & 75.7 & 14.3 & 5.8 \\
\hline 6208018 & 2 & 71.2 & 18.9 & 5.9 \\
\hline 6222530 & 2 & 64.6 & 12.8 & 12.8 \\
\hline 6307132 & 3 & 50.5 & 10.3 & 10.3 \\
\hline 6352407 & 3 & 59.5 & 19.1 & 19.1 \\
\hline 6776494 & 3 & 55.4 & 10.9 & 10.9 \\
\hline 6783217 & 2 & 81.5 & 8.5 & 2.8 \\
\hline 6924074 & 3 & 58.3 & 7.4 & 7.4 \\
\hline 6952783 & 2 & 76.6 & 13.4 & 4.4 \\
\hline 7046554 & 2 & 79.3 & 10.7 & 3.9 \\
\hline 7468195 & 3 & 60.2 & 8.3 & 8.3 \\
\hline 7504619 & 3 & 56.8 & 16.6 & 16.6 \\
\hline 7584122 & 3 & 55.8 & 8.4 & 8.4 \\
\hline 7595722 & 2 & 77.9 & 12.1 & 4.2 \\
\hline 7693845 & 2 & 76.5 & 13.5 & 3.5 \\
\hline 7769544 & 3 & 52.7 & 16.0 & 16.0 \\
\hline 7898594 & 2 & 75.5 & 14.5 & 3.8 \\
\hline 8098454 & 3 & 67.5 & 8.6 & 8.6 \\
\hline 8107355 & 1 & 28.2 & 12.1 & 28.2 \\
\hline
\end{tabular}

Table A.1. continued.

\begin{tabular}{|c|c|c|c|c|}
\hline $\mathrm{KIC}$ & $\begin{array}{c}\text { Number of } \\
\text { rotational } \\
\text { components }\end{array}$ & $i\left(^{\circ}\right)$ & $\sigma_{i,+}\left(^{\circ}\right)$ & $\sigma_{i,-}\left({ }^{\circ}\right)$ \\
\hline 8145017 & 3 & 46.9 & 10.6 & 10.6 \\
\hline 8192753 & 3 & 48.3 & 10.2 & 10.2 \\
\hline 8645227 & 1 & 18.2 & 7.7 & 18.2 \\
\hline 8827367 & 3 & 54.6 & 9.0 & 9.0 \\
\hline 8893299 & 2 & 69.1 & 20.9 & 9.9 \\
\hline 9145781 & 2 & 83.6 & 6.4 & 2.6 \\
\hline 9157260 & 3 & 67.0 & 7.9 & 7.9 \\
\hline 9219983 & 1 & 42.9 & 9.5 & 42.9 \\
\hline 9335457 & 3 & 59.2 & 7.8 & 7.8 \\
\hline 9418101 & 2 & 74.2 & 15.8 & 7.6 \\
\hline 9814077 & 2 & 80.0 & 10.0 & 2.7 \\
\hline 9893437 & 2 & 76.9 & 13.1 & 4.3 \\
\hline 9896174 & 2 & 74.1 & 15.9 & 4.6 \\
\hline 9956184 & 2 & 71.3 & 18.8 & 6.4 \\
\hline 10198496 & 3 & 65.3 & 8.9 & 8.9 \\
\hline 10199289 & 2 & 80.3 & 9.7 & 4.0 \\
\hline 10353556 & 3 & 43.1 & 10.0 & 10.0 \\
\hline 10482211 & 2 & 77.9 & 12.1 & 5.3 \\
\hline 10581491 & 2 & 69.3 & 20.7 & 11.7 \\
\hline 10675916 & 3 & 54.5 & 8.1 & 8.1 \\
\hline 10734124 & 3 & 52.1 & 11.3 & 11.3 \\
\hline 11015392 & 2 & 82.5 & 7.6 & 2.4 \\
\hline 11038809 & 3 & 45.3 & 16.8 & 16.8 \\
\hline 11043770 & 2 & 80.0 & 10.0 & 3.3 \\
\hline 11852899 & 3 & 55.8 & 7.9 & 7.9 \\
\hline 12115374 & 2 & 80.8 & 9.3 & 4.7 \\
\hline 12203197 & 3 & 57.4 & 8.3 & 8.3 \\
\hline
\end{tabular}

Table A.2. Properties of the 21 RGB stars from Corsaro et al. (2017) sample.

\begin{tabular}{lccccc}
\hline \hline KIC & $\begin{array}{c}\text { Number of } \\
\text { rotational } \\
\text { components }\end{array}$ & $i\left(^{\circ}\right)$ & $\sigma_{i,+}\left(^{\circ}\right)$ & $\sigma_{i,-}\left(^{\circ}\right)$ & $\begin{array}{c}\text { Field or } \\
\text { cluster star }\end{array}$ \\
\hline 2437325 & 1 & 39.1 & 17.4 & 39.1 & NGC 6791 \\
2570244 & 1 & 36.3 & 16.0 & 36.3 & NGC 6791 \\
3744043 & 3 & 47.1 & 16.4 & 16.4 & Field \\
5111718 & 2 & 68.1 & 21.9 & 8.4 & NGC 6819 \\
5112072 & 3 & 63.3 & 13.0 & 13.0 & NGC 6819 \\
5113441 & 1 & 37.7 & 16.7 & 37.7 & NGC 6819 \\
6117517 & 1 & 13.0 & 5.4 & 13.0 & Field \\
6144777 & 3 & 63.6 & 12.5 & 12.5 & Field \\
7060732 & 3 & 65.7 & 11.1 & 11.1 & Field \\
7619745 & 2 & 78.1 & 11.9 & 4.8 & Field \\
8366239 & 2 & 73.1 & 8.4 & 8.4 & Field \\
8475025 & 2 & 80.2 & 9.8 & 5.1 & Field \\
8718745 & 1 & 14.7 & 6.2 & 14.7 & Field \\
9267654 & 2 & 72.4 & 17.6 & 6.0 & Field \\
9475697 & 1 & 20.2 & 8.5 & 20.2 & Field \\
9882316 & 1 & 17.6 & 7.4 & 17.6 & Field \\
10123207 & 1 & 13.5 & 5.6 & 13.5 & Field \\
11353313 & 2 & 71.0 & 9.7 & 9.7 & Field \\
11913545 & 2 & 75.4 & 14.6 & 3.7 & Field \\
11968334 & 3 & 54.0 & 14.3 & 14.3 & Field \\
12008916 & 2 & 70.6 & 19.4 & 6.5 & Field \\
\hline & & & & &
\end{tabular}




\section{Appendix B: Examples of oscillation spectra and stretched period échelle diagrams}

The oscillation spectra with the azimuthal order of dipole gravity-dominated mixed modes identified, as well as the corresponding échelle diagrams are presented for:

- KIC 1569842 with the three $m=\{-1,0,1\}$ rotational components observed (Fig. B.1);

- KIC 3098179 with the three $m=\{-1,0,1\}$ rotational components observed (Fig. B.2);

- KIC 1723843 with only the two $m= \pm 1$ rotational components observed (Fig. B.3);

- KIC 2141255 with only the two $m= \pm 1$ rotational components observed (Fig. B.4);

- KIC 2303101 with only the $m=0$ rotational component observed (Fig. B.5);

- KIC 3645589 with only the $m=0$ rotational component observed (Fig. B.6);

- the Kuszlewicz et al. (2019a) star KIC 3531478 with the three $m=\{-1,0,1\}$ rotational components observed for which we are in agreement (Fig. B.7);

- the Kuszlewicz et al. (2019a) star KIC 7504619 with the three $m=\{-1,0,1\}$ rotational components observed for which we are in agreement (Fig. B.8);

- the Kuszlewicz et al. (2019a) star KIC 4731138 with only the two $m= \pm 1$ rotational components observed for which we are in agreement (Fig. B.9);

- the Kuszlewicz et al. (2019a) star KIC 3634488 with only the two $m= \pm 1$ rotational components observed for which we are in marginal agreement (Fig. B.10);

- the Kuszlewicz et al. (2019a) star KIC 4996676 with only the $m=0$ rotational component observed for which we are in agreement (Fig. B.11);

- the Kuszlewicz et al. (2019a) star KIC 3446775 with only the $m=0$ rotational component observed for which we are in agreement (Fig. B.12);

- the Corsaro et al. (2017) NGC 6791 star KIC 2437325 with only the $m=0$ rotational component observed for which we are in agreement (Fig. B.13);

- the Corsaro et al. (2017) NGC 6819 star KIC 5111718 with only the two $m= \pm 1$ rotational components observed for which we are in agreement (Fig. B.14);

- the Corsaro et al. (2017) field star KIC 6117517 with only the $m=0$ rotational component observed for which we are in agreement (Fig. B.15);

- the Corsaro et al. (2017) field star KIC 7060732 with the three $m=\{-1,0,1\}$ rotational component observed for which we are in marginal agreement (Fig. B.16);

- the Corsaro et al. (2017) field star KIC 8475025 with only the two $m= \pm 1$ rotational components observed for which we are in agreement (Fig. B.17).

All these stars lie on the red giant branch.
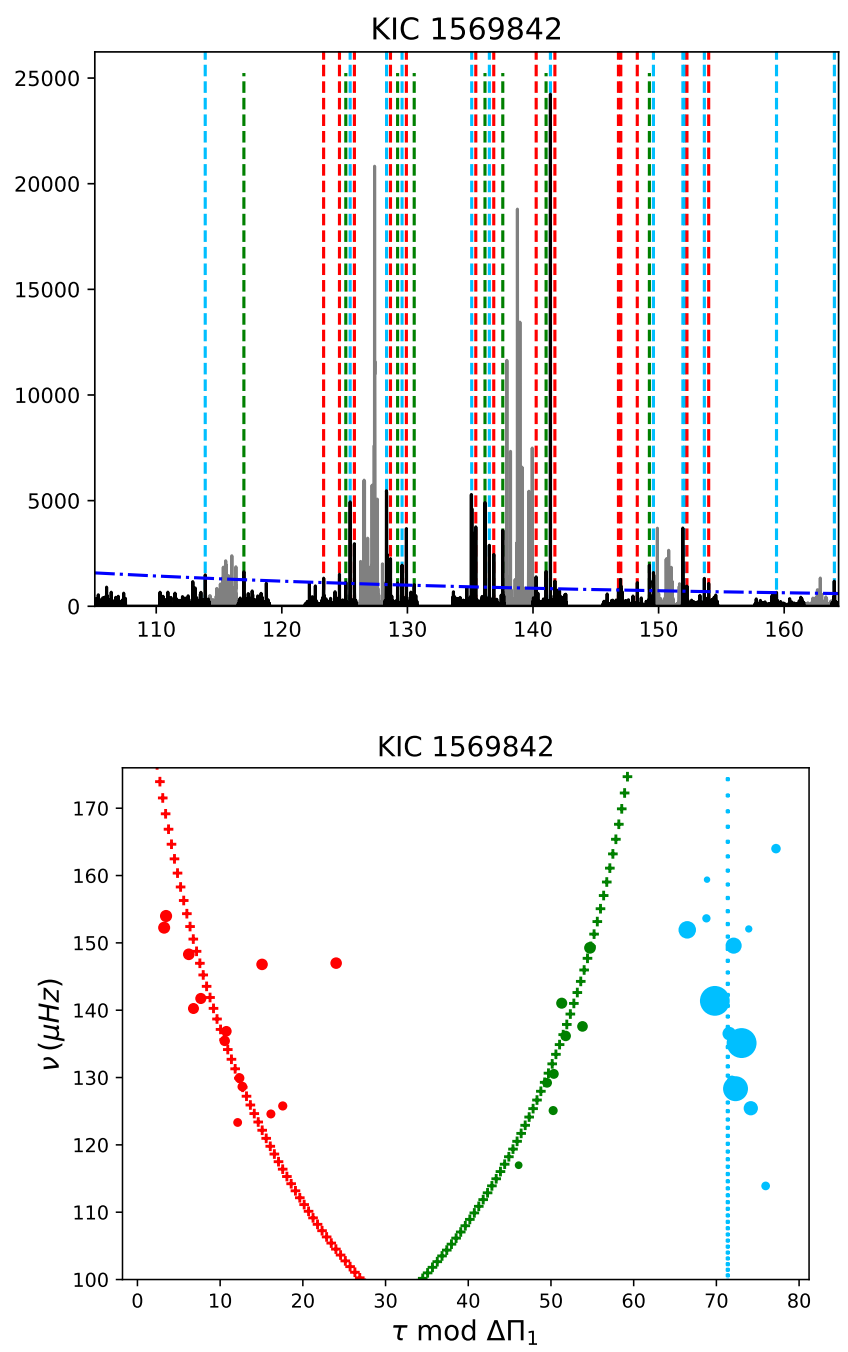

Fig. B.1. KIC 1569842 with three observed rotational components. We found an inclination of $i=32.5 \pm 13.8^{\circ}$. Upper panel: oscillation spectrum where frequency intervals around radial and quadrupole modes have been removed. The horizontal blue dashed-line indicates the level above which oscillation modes are considered as significant. Pressuredominated mixed-modes are in grey, gravity-dominated mixed-modes are in black. Modes with azimuthal orders $m=\{-1,0,1\}$ are indicated by vertical dashed lines in green, light blue and red, respectively. Lower panel: échelle diagram in stretched period with same color code. Observed modes are represented by dots and the symbol size varies as the measured power spectral density. The fit of rotational components are represented by crosses with the color coding the azimuthal order. 

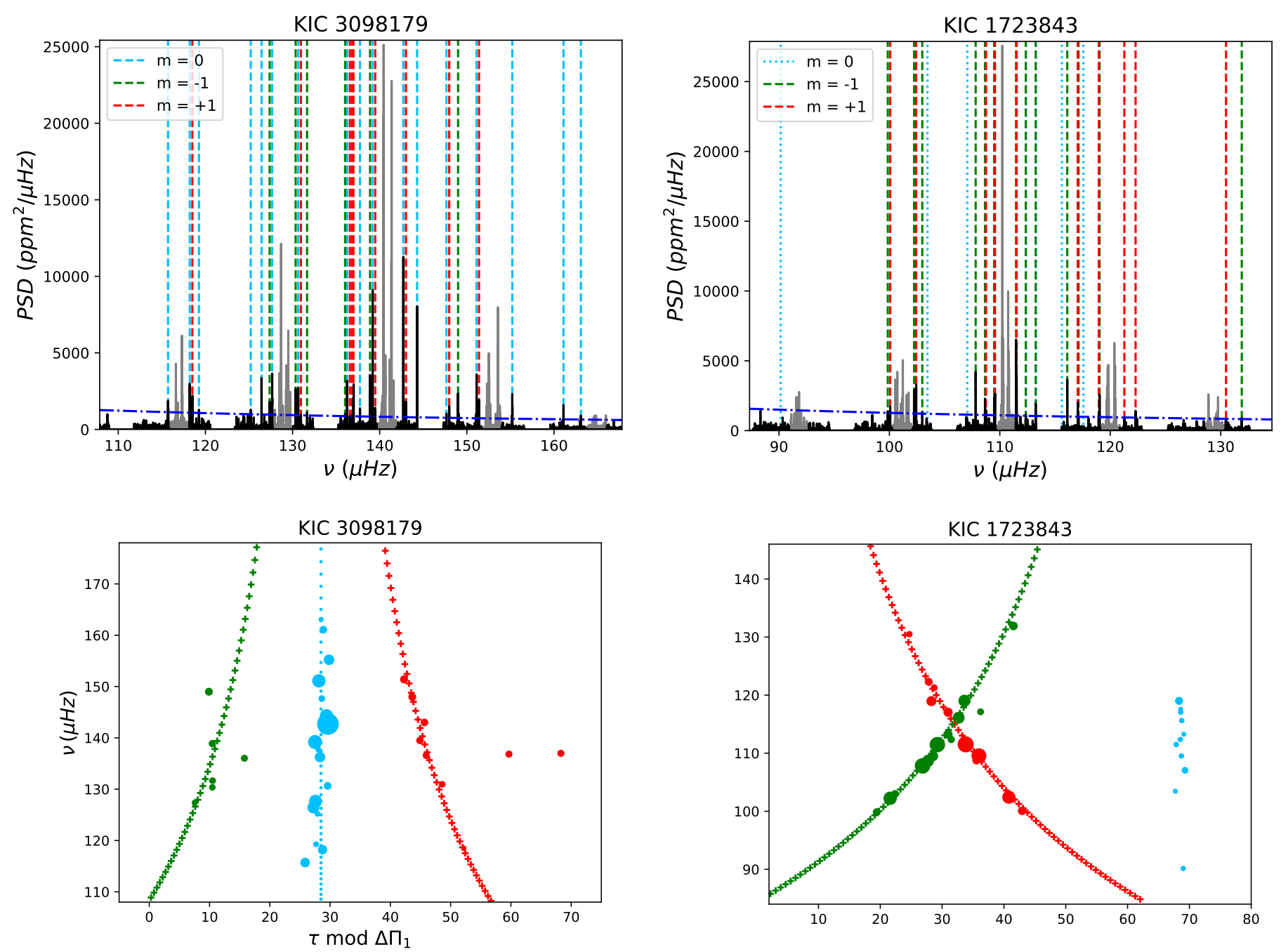

Fig. B.2. KIC 3098179 with three observed rotational components. We found an inclination of $i=39.9 \pm 17.3^{\circ}$.

Fig. B.3. KIC 1723843 with two observed rotational components. Modes with $m=0$ have been identified a posteriori considering that the $m=0$ ridge is median with respect to $m= \pm 1$ ridges in the échelle diagram. We thus represented them by dotted lines in the spectrum to distinguish them from $m= \pm 1$ that are directly visible. We found an inclination of $i=74.3 \pm_{7.2}^{15.7 \circ}$. 
C. Gehan et al.: Automated approach to measure stellar inclinations: validation on the red giant branch

KIC 2141255
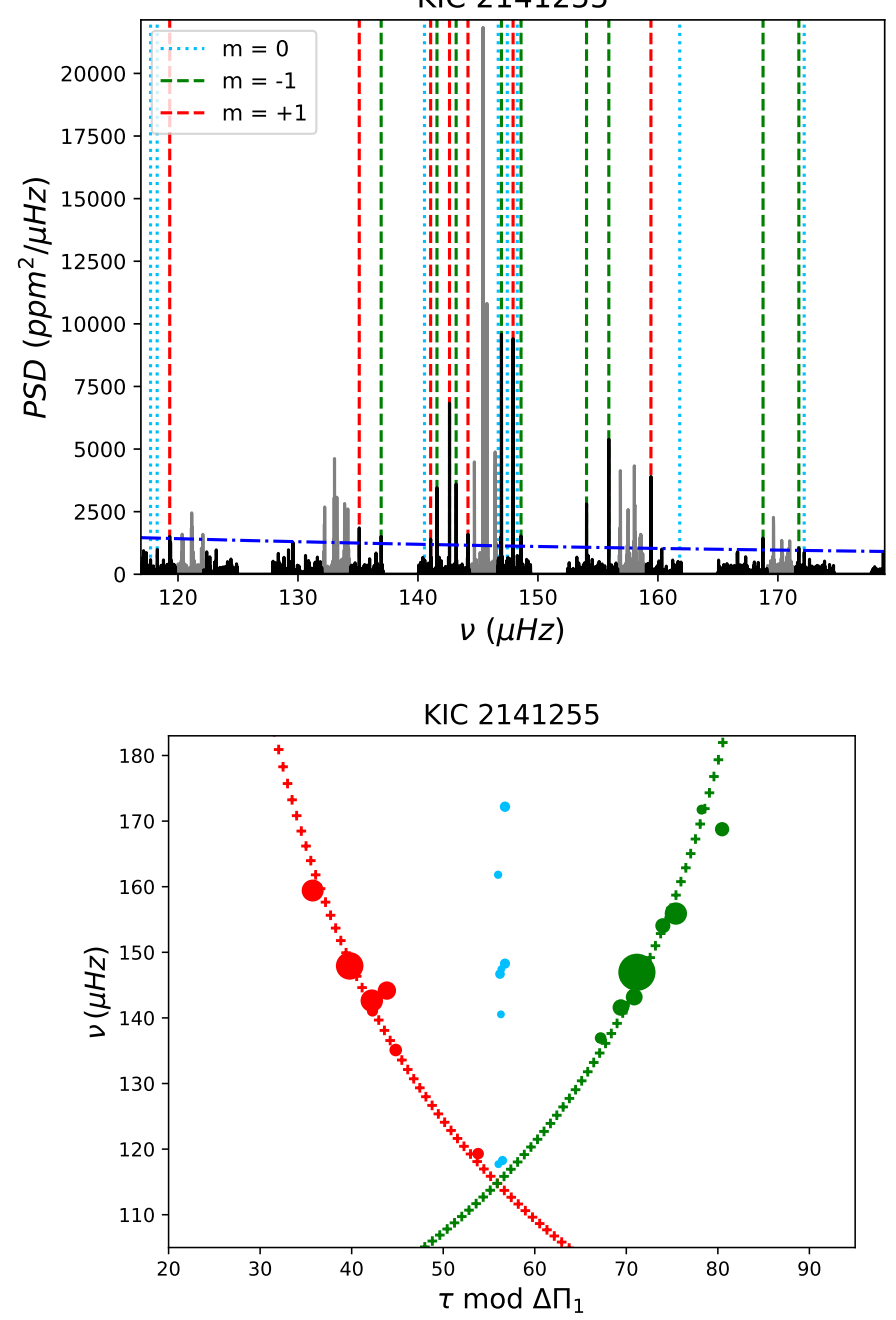

Fig. B.4. KIC 2141255 with two observed rotational components. We found an inclination of $i=76.4 \pm_{8.2}^{13.60}$.
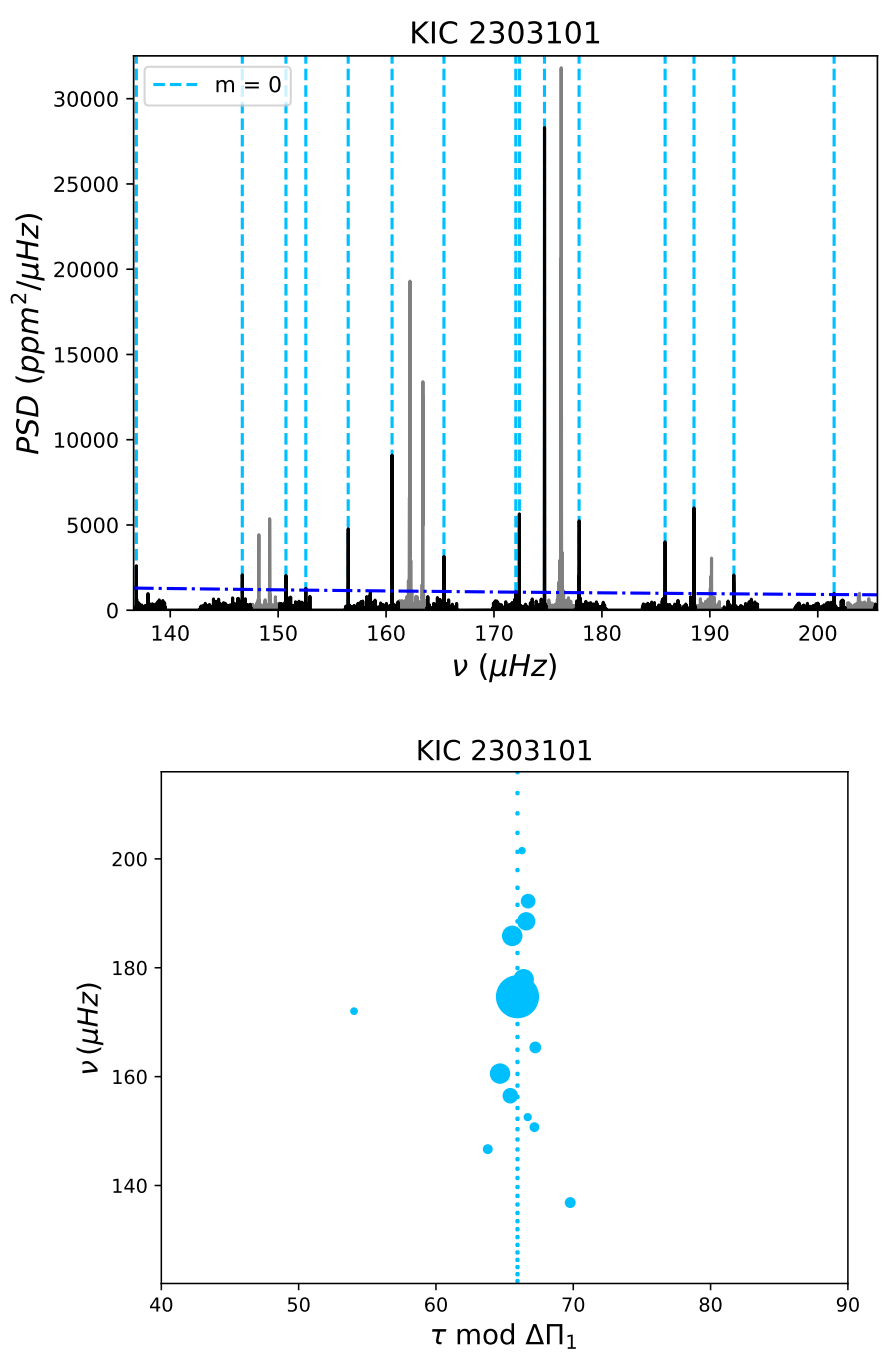

Fig. B.5. KIC 2303101 with one observed rotational component. We found an inclination of $i=19.8 \pm_{19.8}^{8.4 \circ}$. 

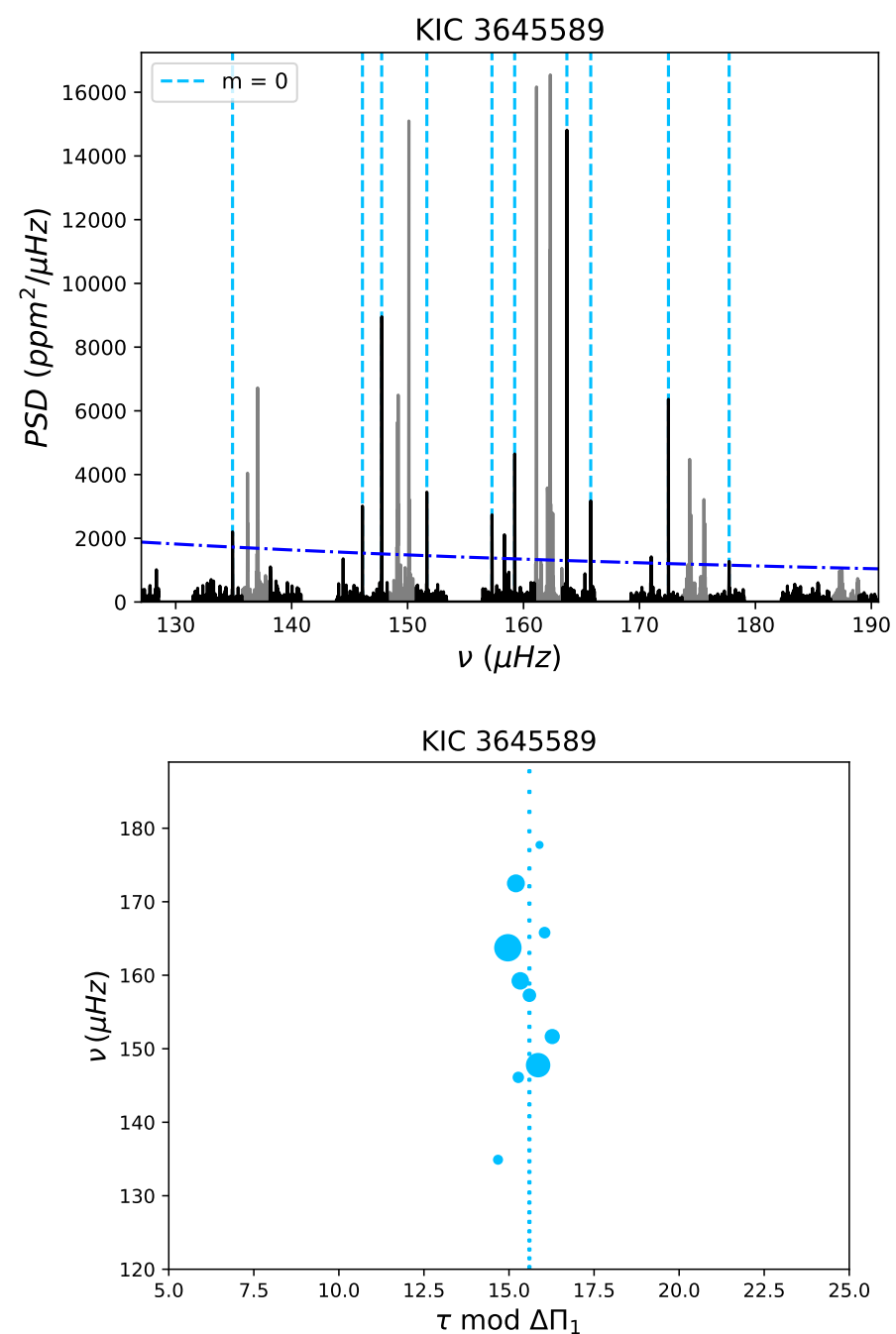

Fig. B.6. KIC 3645589 with one observed rotational component. We found an inclination of $i=18.9 \pm_{18.9}^{8.0 \circ}$.
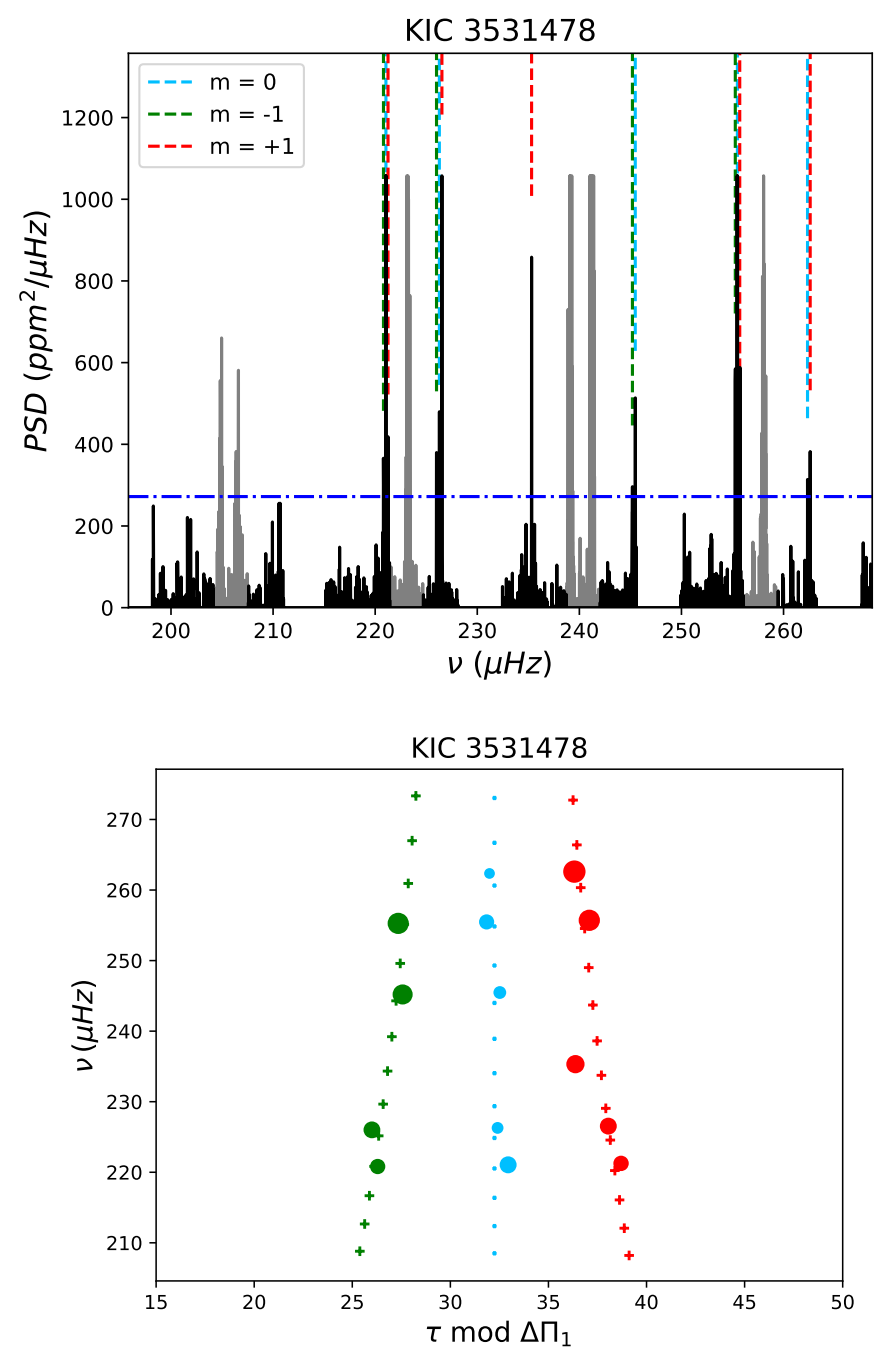

Fig. B.7. Same as Fig. B.1 for KIC 3531478 with three observed rotational components. We found an inclination of $i=41.2 \pm 9.3^{\circ}$ consistent with Kuszlewicz et al. (2019a) measurement of $i=46.8 \pm_{3.1}^{3.2 \circ}$. 
C. Gehan et al.: Automated approach to measure stellar inclinations: validation on the red giant branch
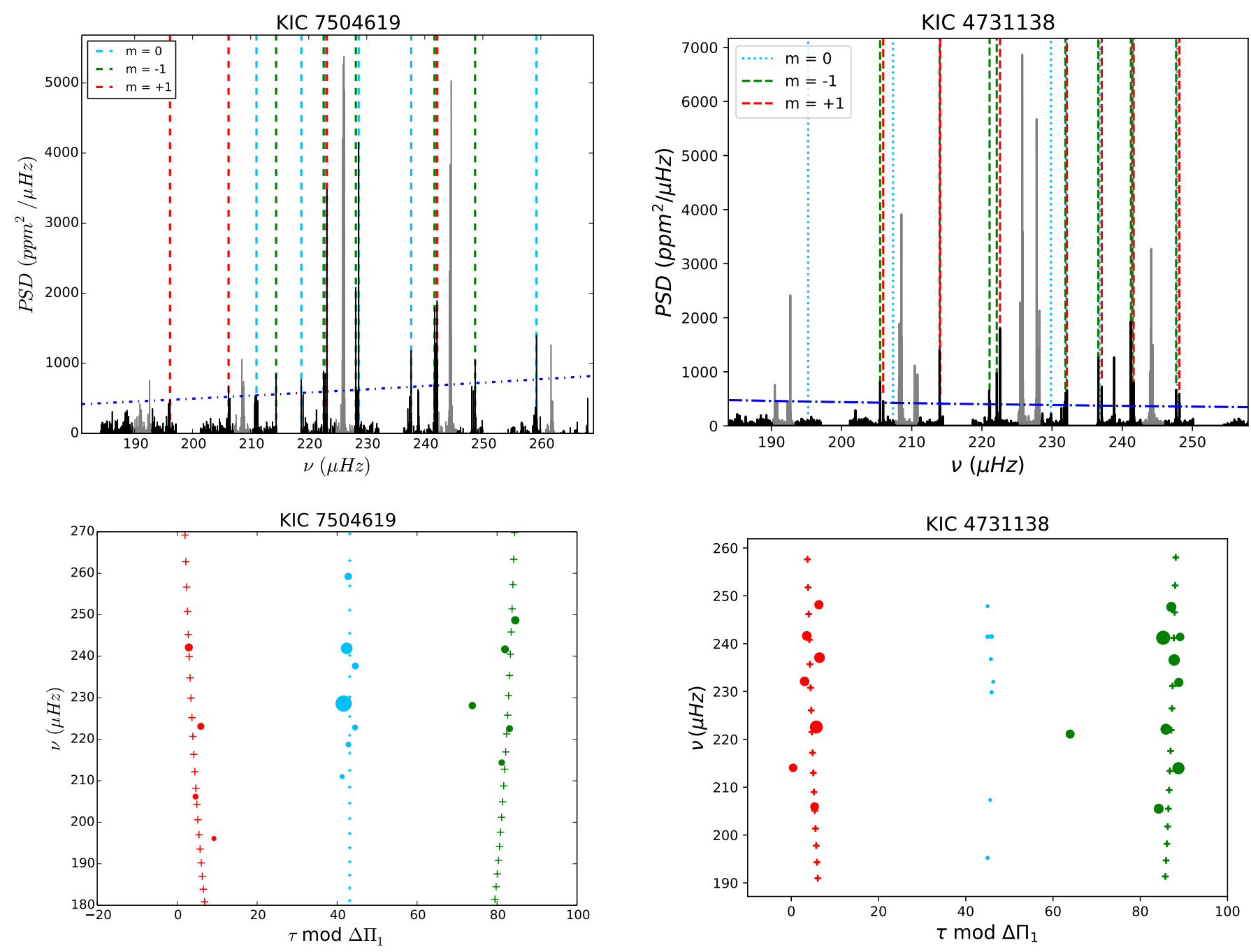

Fig. B.8. Same as Fig. B.1 for KIC 7504619 with three observed rotational components. We found an inclination of $i=56.8 \pm 16.6^{\circ}$ in agreement with Kuszlewicz et al. (2019a) measurement of $i=59.3 \pm_{2.5}^{2.4 \circ}$.

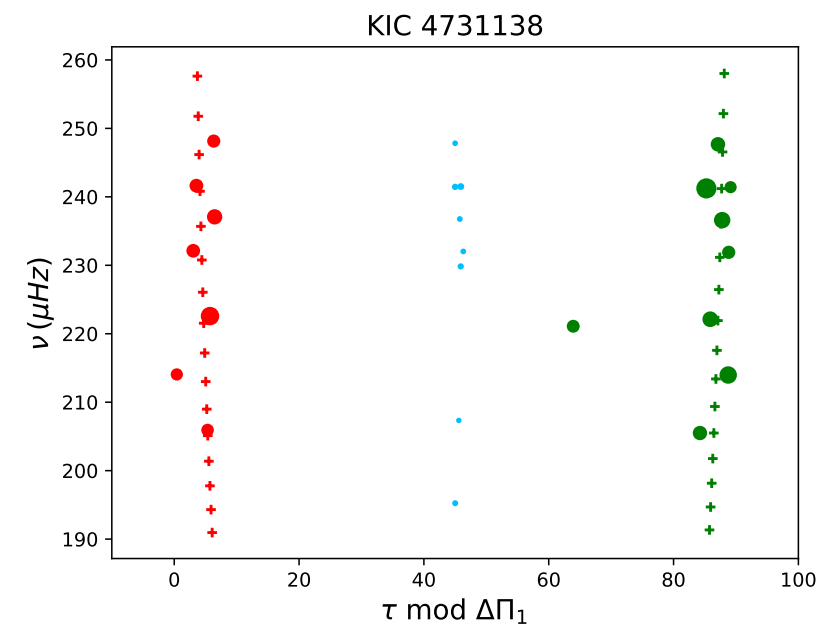

Fig. B.9. Same as Fig. B.1 for KIC 4731138 with two observed rotational components. We found an inclination of $i=79.8 \pm_{31}^{10.2 \circ}$ consistent with Kuszlewicz et al. (2019a) measurement of $i=84.5 \pm_{3.0}^{3.30}$. 
A\&A 645, A124 (2021)
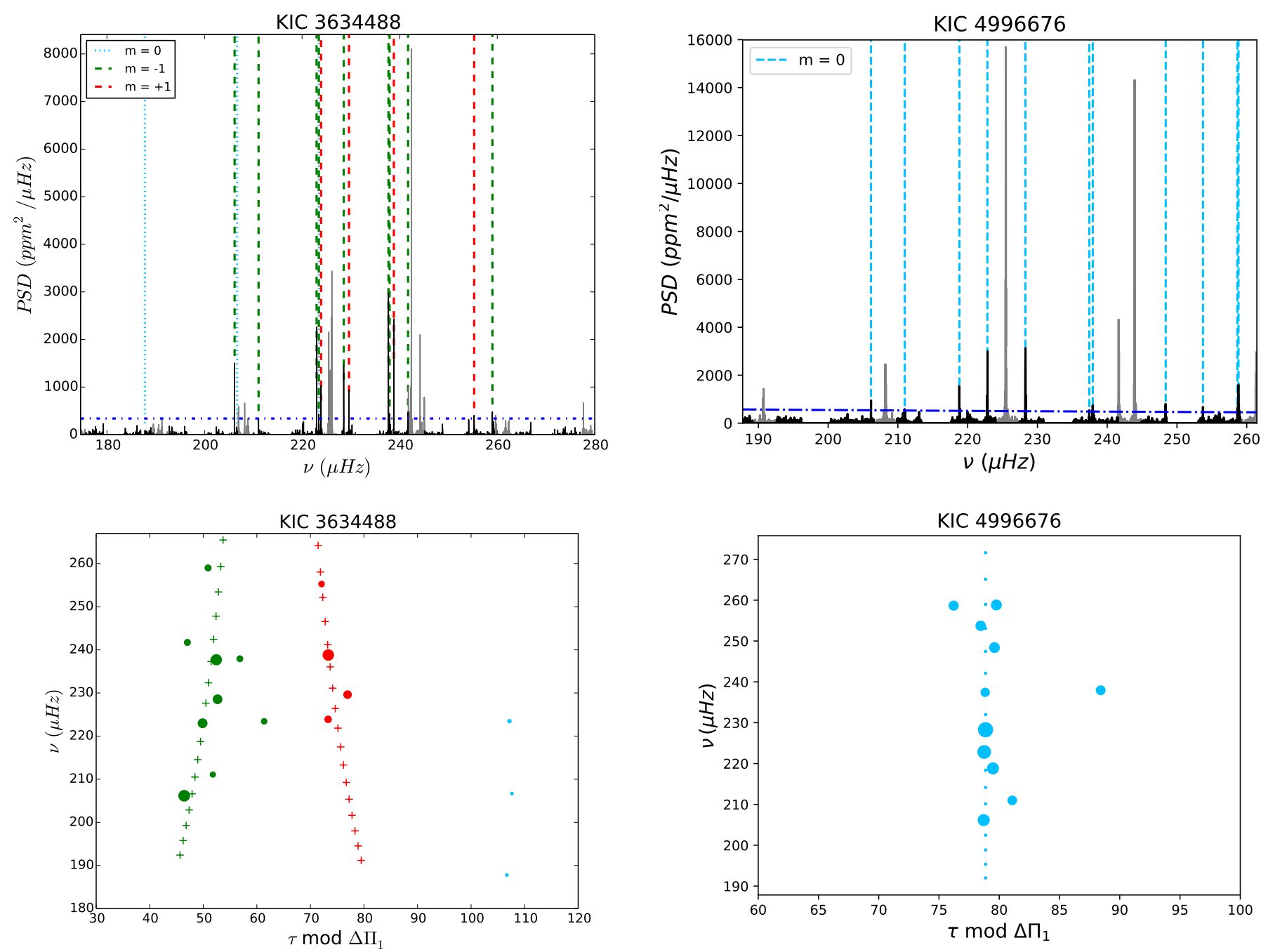

Fig. B.10. Same as Fig. B.1 for KIC 3634488 with two observed rotational components. We found an inclination of $i=86.2 \pm_{9.7}^{3.8 \circ}$ in marginal agreement with Kuszlewicz et al. (2019a) measurement of Fig. B.11. Same as Fig. B.1 for KIC 4996676 with one observed rotational component. We found an inclination of $i=30.1 \pm_{30.1}^{13.0 \circ}$ consistent with Kuszlewicz et al. (2019a) measurement of $i=5.7 \pm_{4.0}^{2.60}$. 
C. Gehan et al.: Automated approach to measure stellar inclinations: validation on the red giant branch
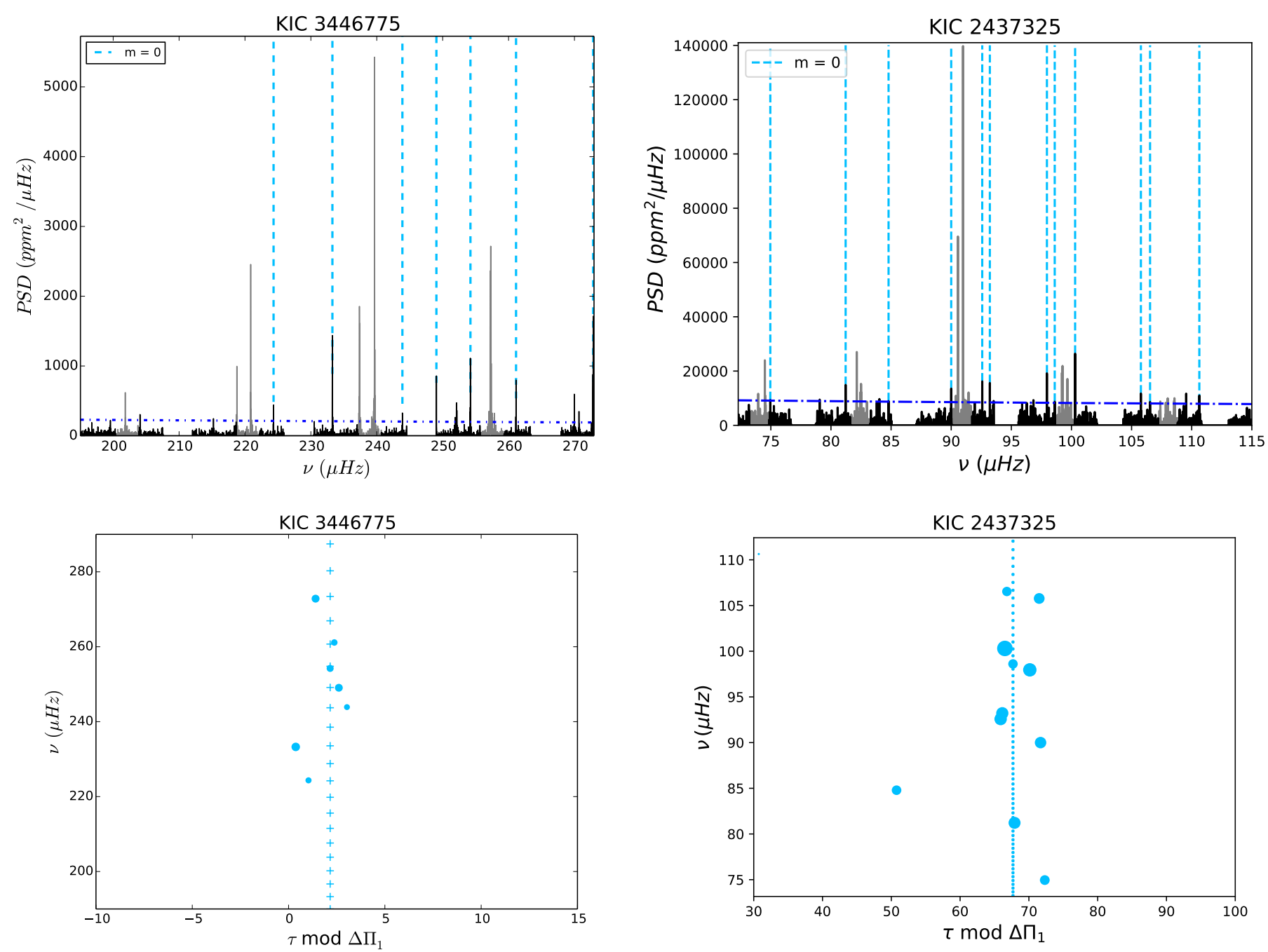

Fig. B.12. Same as Fig. B.1 for KIC 3446775 with one observed rotational component. We found an inclination of $i=31.8 \pm_{31.8}^{13.8}$ in agreement with Kuszlewicz et al. (2019a) measurement of $i=10.3 \pm 4.3^{\circ}$.

Fig. B.13. Same as Fig. B.1 for the NGC 6791 star KIC 2437325 with one observed rotational component. We found an inclination of $i=39.1 \pm_{39.1}^{17.4 \circ}$ consistent with Corsaro et al. (2017) measurement of $i=11 \pm 5^{\circ}$. 

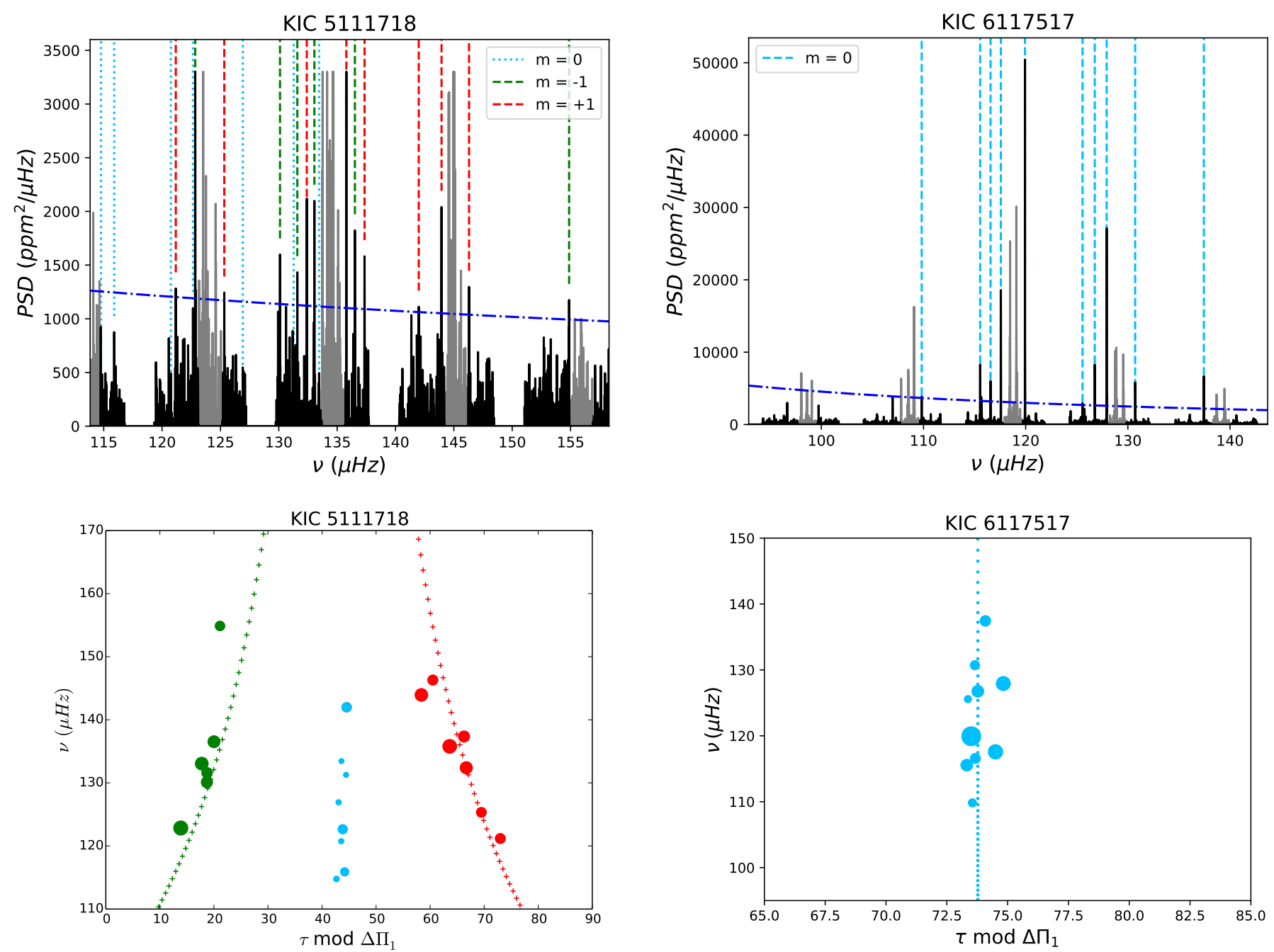

Fig. B.14. Same as Fig. B.1 for the NGC6819 star KIC 5111718 with two observed rotational components. We found an inclination of $i=68.1 \pm_{8.4}^{21.9 \circ}$ consistent with Corsaro et al. (2017) measurement of $i=64 \pm 4^{\circ}$.

Fig. B.15. Same as Fig. B.1 for the field star KIC 6117517 with one observed rotational component. We found an inclination of $i=13.0 \pm_{13.0}^{5.4 \circ}$ consistent with Corsaro et al. (2017) measurement of $i=18 \pm 3^{\circ}$. 
C. Gehan et al.: Automated approach to measure stellar inclinations: validation on the red giant branch
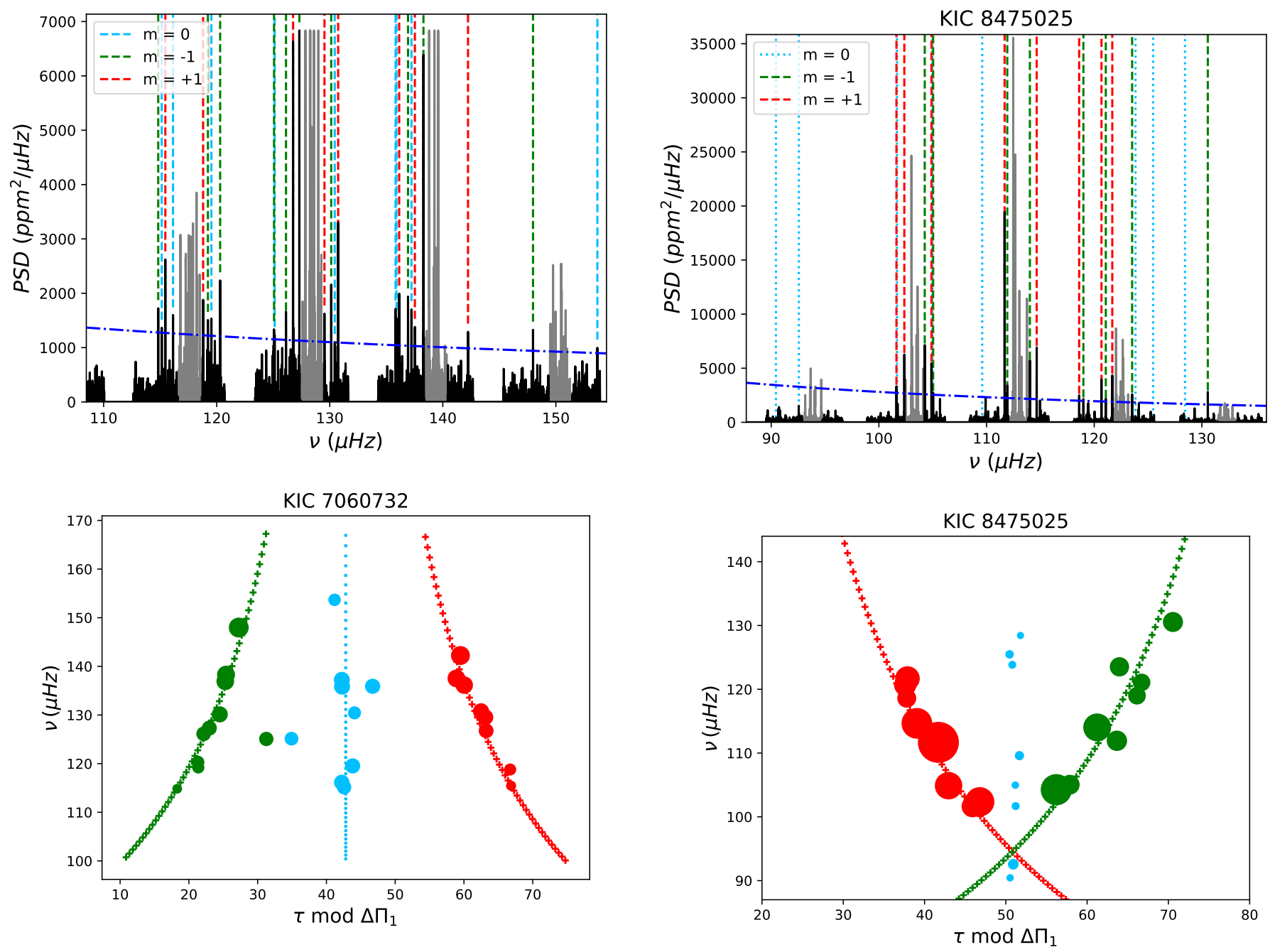

Fig. B.16. Same as Fig. B.1 for the field star KIC 7060732 with three observed rotational components. We found an inclination of $i=65.7 \pm$ $11.1^{\circ}$ marginally consistent with Corsaro et al. (2017) measurement of $i=79 \pm 2^{\circ}$.

Fig. B.17. Same as Fig. B.1 for the field star KIC 8475025 with two observed rotational components. We found an inclination of $i=$ $80.2 \pm_{5.1}^{9.8 \circ}$ consistent with Corsaro et al. (2017) measurement of $i=$ $90 \pm 3^{\circ}$. 